\title{
Shear-thinning in dense colloidal suspensions and its effect on elastic instabilities: from the microscopic equations of motion to an approximation of the macroscopic rheology
}

\author{
Alexandre NICOLAS ${ }^{\mathrm{a}, \mathrm{b}, *}$, Matthias FUCHS ${ }^{\mathrm{c}}$ \\ ${ }^{a}$ Université Grenoble-Alpes, LIPhy, F-38000 Grenoble, France \\ ${ }^{b}$ CNRS, LIPhy, F-38000 Grenoble, France \\ ${ }^{c}$ Fachbereich Physik, Universität Konstanz, 78457 Konstanz, Germany
}

\begin{abstract}
In the vicinity of their glass transition, dense colloidal suspensions acquire elastic properties over experimental timescales. We investigate the possibility of a visco-elastic flow instability in curved geometry for such materials. To this end, we first present a general strategy extending a first-principles approach based on projections onto slow variables (so far restricted to strictly homogeneous flow) in order to handle inhomogeneities. In particular, we separate the advection of the microstructure by the flow, at the origin of a fluctuation advection term, from the intrinsic dynamics. On account of the complexity of the involved equations, we then opt for a drastic simplification of the theory, in order to establish its potential to describe instabilities. These very strong approximations lead to a constitutive equation of the White-Metzner class, whose parameters are fitted with experimental measurements of the macroscopic rheology of a glass-forming colloidal dispersion. The model properly accounts for the shear-thinning properties of the dispersions, but, owing to the approximations, the description is not fully quantitative. Finally, we perform a linear stability analysis of the flow in the experimentally relevant cylindrical (Taylor-Couette) geometry and provide evidence that shear-thinning strongly stabilises the flow, which can explain why visco-elastic instabilities are not observed in dense colloidal suspensions.
\end{abstract}

Keywords: rheology; dense colloidal suspensions; mode-coupling theory; viscoelastic instability

\footnotetext{
*Corresponding author's email: alexandre.nicolas@polytechnique.edu; Phone: $(+54) 9.2944 .81 .9476$.

Present address: CONICET \& Centro Atómico Bariloche, Bariloche, Argentina.
} 


\section{Introduction}

\subsection{Observations}

Take a small amount of carbon black powder and disperse it into water: this gives pigmented ink. From a rheological perspective, it is a colloidal suspension which flows similarly to water, albeit with a somewhat higher viscosity. But this Newtonian behaviour, which holds generically for very dilute suspensions, is strongly altered when the volume fraction $\phi$ of colloids gets larger. Most strikingly, the viscosity and relaxation time of the suspension increase dramatically when $\phi$ approaches a "critical" packing fraction $\phi_{g}\left(\phi_{g} \approx 0.56\right.$ for hard-sphere-like colloids [1]). For $\phi \gtrsim \phi_{g}$, the material retains elastic properties over any experimental timescale, in a fashion reminiscent of the emergence of glassiness in supercooled melts of some metallic alloys, when the temperature declines [2]. Despite these dramatic changes, the structure of the material remains essentially liquid-like throughout the transition. Accordingly, it appears sensible to compare the rheology of very dense colloidal suspensions to that of other visco-elastic liquids. In particular, one may wonder why a variety of complex fluids among the latter, such as worm-like micelles or polymer solutions [3, 44], are prone to a (non-inertial) flow instability in curved geometry, leading for instance to the formation of vortices, while, to the best of our knowledge, no such visco-elastic instability has ever been reported in very dense colloidal suspensions.

\subsection{A microscopic approach using mode-coupling theory}

The level of difficulty required to rationalise the rheology of suspensions strongly depends on the volume fraction $\phi$ of interest. In the dilute regime, the fluid is Newtonian; its viscosity is independent of the applied shear rate. More quantitatively, the linear corrections to the solvent viscosity due to the colloids were worked out by Einstein a little more than a century ago, under the assumption of non-interacting colloids [5]. By a detailed study of the probability distribution function of particle pairs, the approach was extended to interacting colloids by Batchelor and others [6], and led to a description of the semi-dilute regime [7]. For $\phi \approx \phi_{g}$, collective effects become paramount, in that glassiness can be thought of as the entrapment of particles in the "cages" formed by their neighbours; these effects turn a firstprinciples derivation of the macroscopic rheology into a formidable challenge, all the more so as the presence of flow distorts the structure of the material 
away from its "quiescent configuration" and gives rise to complex interplays [8].

Nevertheless, at the expense of some uncontrolled approximations, the mode-coupling theory developed by Sjögren, Bengtzelius, Götze, Sjolander, and others [9, 10] succeeded in rationalising the phenomenology of the glass transition by focusing on the evolution and the relaxation of the (slow) density modes of the system and on their coupling to the other (faster) variables. In the last decade, Fuchs, Cates et al. [11, 12], and Miyazaki et al. [13] in a parallel endeavour, were able to extend this framework to situations of flow, in which the colloids are dragged by a prescribed solvent flow. The state of the art of this theory encompasses arbitrary, potentially time-dependent incompressible solvent flows, in two or three dimensions [12, 14].

However, the derivation hinges on the assumption of a perfectly homogeneous flow throughout space; this hampers the investigation of any flow instability. Indeed, perturbations, which break homogeneity, are not handled adequately; in particular, the mechanism describing their (expected) advection with the flow is still missing in the equations. Moreover, the complexity of the final equations giving the stress as a function of the strain history is a deterrent to any stability analysis in non-trivial geometry.

\subsection{Objectives of the article}

In this contribution, we first propose a general way to extend the formalism and handle flow inhomogeneities, insisting in particular on the recovery of a fluctuation advection term and on the limit of locally homogeneous flow. Then, we follow the endeavour pioneered in Ref. [15] to reduce the final equations to a tractable constitutive equation. This will come at the expense of very strong (but explicitly exposed) approximations and clearly undermine the accuracy of the description. Nevertheless, the ensuing simple model, which falls in the White-Metzner class [16], will allow us to capture the experimentally measured low-shear-rate rheology and high-shear-rate rheology in a model colloidal glass-forming dispersion [17, 18]. Finally, a linear stability analysis of the flow will be performed, in cylindrical (Taylor-Couette) geometry and the (stabilising) effect of effect of shear-thinning on the viscoelastic flow will be numerically assessed.

\section{Theoretical probabilistic framework}

We start by presenting the theoretical underpinning of the rheological equations that extend quiescent mode-coupling theory. 
Let us consider an assembly of $N$ colloidal particles dispersed in a solvent and evolving by Brownian motion in a volume $V$, for instance with periodic boundary conditions.

\subsection{From the overdamped Langevin equation to the Smoluchowski equation}

To describe the microscopic motion of particle $i \in\{1, \ldots, N\}$, we posit an overdamped Langevin equation acting on its velocity $\dot{\boldsymbol{r}}_{\boldsymbol{i}}$ :

$$
\zeta\left[\dot{\boldsymbol{r}}_{\boldsymbol{i}}-\boldsymbol{v}^{\mathrm{solv}}\left(\boldsymbol{r}_{\boldsymbol{i}}\right)\right]=\boldsymbol{F}_{\boldsymbol{i}}+\boldsymbol{f}_{\boldsymbol{i}}^{\text {th }}
$$

Here, $\boldsymbol{F}_{\boldsymbol{i}}$ is the conservative force that derives from the global potential energy of the system, and the $\boldsymbol{f}_{\boldsymbol{i}}{ }^{\text {th }}$ 's are random Gaussian thermal fluctuations, viz., $\left\langle\boldsymbol{f}_{\boldsymbol{i}}^{\text {th }}(t)\right\rangle=\mathbf{0}$ and $\left\langle\boldsymbol{f}_{\boldsymbol{i}}^{\text {th }}(t) \otimes \boldsymbol{f}_{\boldsymbol{j}}{ }^{\text {th }}\left(t^{\prime}\right)\right\rangle=2 k_{B} T \zeta \delta_{i j} \delta\left(t-t^{\prime}\right) \mathbb{I}$, where $\mathbb{I}$ is the identity matrix in $d$ dimensions. The frictional force on the left-hand side (lhs) involves a frictional coefficient $\zeta$ and the particle velocity relative to the (prescribed) local solvent velocity $\boldsymbol{v}^{\text {solv }}(\boldsymbol{r})$; for an incompressible flow, the latter should satisfy $\nabla \cdot \boldsymbol{v}^{\text {solv }}=0$. Hydrodynamic effects, being presumably subordinate to short-range interactions in dense systems, are neglected.

Rather than focusing on the motion of individual particles, we adopt a statistical approach. Equation 1 is recast into the following equations for the evolution of the probability $\psi(\Gamma ; t)$ to find the system in the microscopic configuration $\Gamma \equiv\left(\boldsymbol{r}_{1}, \ldots, \boldsymbol{r}_{\boldsymbol{N}}\right)$ at time $t[19]$ :

$$
\begin{cases}\psi(\Gamma ; t=0) & =\psi_{0}(\Gamma) \\ \partial_{t} \psi(\Gamma ; t) & =\Omega(\Gamma ; t) \psi(\Gamma ; t) .\end{cases}
$$

Time evolution is given by the Smoluchowski operator

$$
\Omega(\Gamma ; t) \equiv \sum_{i=1}^{N} \partial_{i} \cdot\left[\partial_{i}-\boldsymbol{F}_{\boldsymbol{i}}(\Gamma)-\boldsymbol{v}^{\mathrm{solv}}\left(\boldsymbol{r}_{\boldsymbol{i}}, t\right)\right],
$$

where $\partial_{i} \equiv \frac{\partial}{\partial \boldsymbol{r}_{\boldsymbol{i}}}$ and we have used dimensionless units by setting $\zeta=1$ and $k_{B} T=1$. Here, contrary to Ref. [11, 20, 15, 12], the initial probability density $\psi_{0}(\Gamma)$ need not be the equilibrium distribution: the system can be prepared in an arbitrary configuration.

Equation 2 is formally solved by

$$
\psi(\Gamma ; t)=e_{+}^{\int_{0}^{t} \Omega(\Gamma ; s) d s} \psi_{0}(\Gamma),
$$


where $e_{+}$is a time-ordered product (see Appendix A of Ref. [12]). At time $t$, the evaluation of a many-body function $g$ reads

$$
\langle g\rangle_{t} \equiv \int g(\Gamma) \psi(\Gamma ; t) d \Gamma
$$

Instead of having the probability distribution $\psi$ evolve in time, as in Eq. 3, a dual formulation is sometimes preferable, in which (by means of a partial integration of Eq. (4) $\psi$ is kept constant and the definiton of $g$ evolves with time, analogously to the switch from a wavefunction-evolving Schrödinger representation to an operator-evolving Heisenberg representation in Quantum Mechanics, viz.,

$$
\begin{cases}\psi(\Gamma ; t) & =\psi_{0}(\Gamma) \\ \partial_{t} g(\Gamma ; t) & =\Omega^{\dagger}(\Gamma ; t) g(\Gamma ; t),\end{cases}
$$

where $\Omega^{\dagger}(\Gamma ; t) \equiv \sum_{i=1}^{N}\left[\partial_{i}+\boldsymbol{F}_{\boldsymbol{i}}(\Gamma)+\boldsymbol{v}^{\text {solv }}\left(\boldsymbol{r}_{\boldsymbol{i}}, t\right)\right] \cdot \partial_{i}$ is the adjoint of the Smoluchowski operator, with the formal solution

$$
g(\Gamma ; t)=e_{-}^{\int_{0}^{t} \Omega^{\dagger}(\Gamma ; s) d s} g(\Gamma ; 0),
$$

where $e_{-}$denotes the negatively ordered exponential [12].

\subsection{Auxiliary frame and recovery of an advection term}

Microscopic observables depend on space, via their point of evaluation $\boldsymbol{r}$ : $g(\Gamma ; t) \rightarrow g(\boldsymbol{r} ; \Gamma ; t)$. But the prescribed velocity field generally differs from zero at $\boldsymbol{r}$, so that the evolution of $g(\boldsymbol{r})$ mingles an intrinsic evolution of the system and an advection by the flow field. In previous studies, for instance, Ref. [12], the consideration of a strictly homogeneous system (with vanishing spatial gradients) rendered a disentanglement of the two effects unnecessary and no advection term appeared in the equations. Yet, in the presence of any heterogeneity, such term is expected on physical grounds and is crucial for the study of perturbations, hence, instabilities. Here, we purport to carefully establish its recovery.

To disentangle advection and intrinsic dynamics, it is helpful to observe the dynamics in a frame that moves with the solvent velocity at the point $\boldsymbol{r}_{O}$ and time $t_{o}$ that will be of interest. Thus, we introduce new, time-dependent coordinates

$$
\boldsymbol{r}^{\prime}[\boldsymbol{r}, t] \equiv \boldsymbol{r}-\left(\boldsymbol{r}_{o}(t)-\boldsymbol{r}_{o}\right),
$$

with the backward transform 


$$
\boldsymbol{r}\left[\boldsymbol{r}^{\prime}, t\right]=\boldsymbol{r}^{\prime}+\left(\boldsymbol{r}_{o}(t)-\boldsymbol{r}_{o}\right),
$$

where $\boldsymbol{r}_{o}(t)$ is the pathline of the (non-singular) solvent velocity field $\boldsymbol{v}^{\text {solv }}(\boldsymbol{r}, t)$ that ends at $\boldsymbol{r}_{o}$ at time $t_{o}$, i.e.,

$$
\left\{\begin{array}{l}
\partial_{t} \boldsymbol{r}_{o}(t)=\boldsymbol{v}^{\mathrm{solv}}\left(\boldsymbol{r}_{o}(t), t\right) \\
\boldsymbol{r}_{o}\left(t_{o}\right)=\boldsymbol{r}_{o}
\end{array}\right.
$$

(In Appendix A, we propose an equivalent, alternative approach, rooted in operator formalism rather than change of frame). At a fixed point $\boldsymbol{r}^{\prime}$ in the new frame, $g$ evolves with time as follows:

$$
\begin{aligned}
D_{t} g\left(\boldsymbol{r}\left[\boldsymbol{r}^{\prime}, t\right], \Gamma ; t\right) & \equiv \lim _{d t \rightarrow 0} \frac{g\left(\boldsymbol{r}\left[\boldsymbol{r}^{\prime}, t+d t\right], \Gamma ; t+d t\right)-g\left(\boldsymbol{r}\left[\boldsymbol{r}^{\prime}, t\right], \Gamma ; t\right)}{d t} \\
& =\Omega^{\dagger}(\Gamma ; t) g\left(\boldsymbol{r}\left[\boldsymbol{r}^{\prime}, t\right], \Gamma ; t\right)+\partial_{t} \boldsymbol{r}_{o}(t) \cdot \partial_{\boldsymbol{r}} g\left(\boldsymbol{r}\left[\boldsymbol{r}^{\prime}, t\right], \Gamma ; t\right) \\
& =\Omega^{\dagger}(\Gamma ; t) g\left(\boldsymbol{r}\left[\boldsymbol{r}^{\prime}, t\right], \Gamma ; t\right)+\boldsymbol{v}^{\mathrm{solv}}\left(\boldsymbol{r}_{o}(t), t\right) \cdot \partial_{\boldsymbol{r}} g\left(\boldsymbol{r}\left[\boldsymbol{r}^{\prime}, t\right], \Gamma ; t\right) .
\end{aligned}
$$

Next, we notice that commonly used observables, such as the stress or the density, do not depend intrinsically on space, i.e., there exists a function $\tilde{g}$ such that $g(\boldsymbol{r}, \Gamma) \equiv g\left(\boldsymbol{r}, \boldsymbol{r}_{1}, \ldots, \boldsymbol{r}_{\boldsymbol{N}}\right)=\tilde{g}\left(\boldsymbol{r}_{\mathbf{1}}-\boldsymbol{r}, \ldots, \boldsymbol{r}_{\boldsymbol{N}}-\boldsymbol{r}\right)$. Consequently,

$$
\begin{aligned}
\partial_{\boldsymbol{r}} g(\boldsymbol{r}, \Gamma) & =\partial_{\boldsymbol{r}} \tilde{g}\left(\boldsymbol{r}_{\mathbf{1}}-\boldsymbol{r}, \ldots, \boldsymbol{r}_{\boldsymbol{N}}-\boldsymbol{r}\right) \\
& =-\sum_{i} \partial_{i} \tilde{g}\left(\boldsymbol{r}_{\mathbf{1}}-\boldsymbol{r}, \ldots, \boldsymbol{r}_{\boldsymbol{N}}-\boldsymbol{r}\right) \\
& =-\sum_{i} \partial_{i} g(\boldsymbol{r}, \Gamma) .
\end{aligned}
$$

Inserting this result into Eq. 9, we get

$$
\begin{gathered}
D_{t} g\left(\boldsymbol{r}\left[\boldsymbol{r}^{\prime}, t\right], \Gamma ; t\right)=\left[\sum_{i=1}^{N}\left[\partial_{i}+\boldsymbol{F}_{\boldsymbol{i}}(\Gamma)+\boldsymbol{v}^{\mathrm{solv}}\left(\boldsymbol{r}_{\boldsymbol{i}}, t\right)\right] \cdot \partial_{i}\right] g\left(\boldsymbol{r}\left[\boldsymbol{r}^{\prime}, t\right], \Gamma ; t\right) \\
-\boldsymbol{v}^{\mathrm{solv}}\left(\boldsymbol{r}_{o}(t), t\right) \cdot \sum_{i} \partial_{i} g\left(\boldsymbol{r}\left[\boldsymbol{r}^{\prime}, t\right], \Gamma ; t\right) \\
=\left[\sum_{i=1}^{N}\left[\partial_{i}+\boldsymbol{F}_{\boldsymbol{i}}(\Gamma)+\left(\boldsymbol{v}^{\mathrm{solv}}\left(\boldsymbol{r}_{\boldsymbol{i}}, t\right)-\boldsymbol{v}^{\mathrm{solv}}\left(\boldsymbol{r}_{o}(t), t\right)\right)\right] \cdot \partial_{i}\right] g\left(\boldsymbol{r}\left[\boldsymbol{r}^{\prime}, t\right], \Gamma ; t\right)
\end{gathered}
$$

Denoting by a prime the functions expressed in the new frame, i.e., $f^{\prime}\left(\boldsymbol{r}^{\prime}[\boldsymbol{r}, t], t\right)=f(\boldsymbol{r}, t)$ for a generic function $f$, and remarking that coordinates in the original and new frame are in a one-to-one correspondence, we arrive at 


$$
\begin{aligned}
D_{t} g\left(\boldsymbol{r}\left[\boldsymbol{r}^{\prime}, t\right], \Gamma ; t\right) & \equiv \partial_{t} g^{\prime}\left(\boldsymbol{r}^{\prime}, \Gamma^{\prime} ; t\right) \\
& =\Omega^{\dagger \prime}\left(\Gamma^{\prime}, t\right) g^{\prime}\left(\boldsymbol{r}^{\prime}, \Gamma ; t\right),
\end{aligned}
$$

for any $\boldsymbol{r}^{\prime}$ in the domain, where

$$
\Omega^{\dagger \prime}\left(\Gamma^{\prime}, t\right) \equiv \sum_{i=1}^{N}\left[\partial_{i}^{\prime}+\boldsymbol{F}_{\boldsymbol{i}}{ }^{\prime}\left(\Gamma^{\prime}\right)+\boldsymbol{v}^{\prime}\left(\boldsymbol{r}_{\boldsymbol{i}}{ }^{\prime}, t\right)\right] \cdot \partial_{i}^{\prime}
$$

and

$$
\boldsymbol{v}^{\prime}\left(\boldsymbol{r}^{\prime}, t\right) \equiv \boldsymbol{v}^{\text {solv } \prime}\left(\boldsymbol{r}^{\prime}, t\right)-\boldsymbol{v}^{\text {solv } \prime}\left(\boldsymbol{r}_{o}, t\right) .
$$

Thus, an observable $g^{\prime}$ evaluated at fixed position in the auxiliary frame displays dynamics identical to those of its counterpart $g$ in the original frame (see Eq. 5), except that the velocity field $\boldsymbol{v}^{\text {solv }}$ entering the Smoluchowski operator for $g$ is replaced by a new field $\boldsymbol{v}^{\prime}$ for $g^{\prime}$, which vanishes at $\boldsymbol{r}_{o}$. Using Eq. 13, we see that the evolutions in the two frames are related by

$$
\left.\partial_{t} g^{\prime}\left(\boldsymbol{r}^{\prime}, \Gamma^{\prime} ; t\right)\right|_{\boldsymbol{r}^{\prime}=\boldsymbol{r}^{\prime}[\boldsymbol{r}, t]}=\partial_{t} g(\boldsymbol{r}, \Gamma ; t)+\boldsymbol{v}^{\mathrm{solv}}\left(\boldsymbol{r}_{o}(t), t\right) \cdot \partial_{\boldsymbol{r}} g(\boldsymbol{r}, \Gamma ; t) .
$$

What is the advantage of switching to the coordinates in the auxiliary, then? First, for any (potentially time-dependent) evaluation point $\boldsymbol{r}^{\prime}$, the new Smoluchowski operator $\Omega^{\dagger^{\prime}}\left(\Gamma^{\prime}, t\right)$ is insensitive to global, potentially time-dependent translations in the original frame, i.e., offsets of the velocity field $\boldsymbol{v}^{\text {solv }}\left(\boldsymbol{r}_{\boldsymbol{i}}, t_{1}\right)$. Accordingly, it only depends on the velocity gradient

$\kappa_{\alpha \beta}(\boldsymbol{r}, t) \equiv \partial_{\beta} v_{\alpha}^{\text {solv }}(\boldsymbol{r}, t)=\partial_{\beta}^{\prime} v_{\alpha}^{\prime}\left(\boldsymbol{r}^{\prime}[\boldsymbol{r}, t], t\right)$, responsible for the deformation of the structure. A second considerable benefit emerges for the specific point of evaluation $\boldsymbol{r}^{\prime}=\boldsymbol{r}_{o}$, where the field $\boldsymbol{v}^{\prime}$ vanishes at all times. (Note that in the original frame this point moves as time passes). Then, in Eq. 14, the effects of advection and of the intrinsic dynamics are clearly separated: the latter are reflected by the evolution of $g^{\prime}$ at the point $\boldsymbol{r}^{\prime}=\boldsymbol{r}_{o}$ in the auxiliary frame, where there is no flow, while the second term on the right-hand side (rhs) is the desired advection term; let us once again emphasise that this non-local term is physically crucial in a heterogeneous system.

\subsection{Leading-order locally homogeneous flow}

Formally, Eq. 6 conveys the impression that a microscopic observable $g$, albeit evaluated at a given point $\boldsymbol{r}_{o}$ and time $t_{o}$, depends on the configuration $\Gamma$ of all particles throughout space, and not only at $\boldsymbol{r}=\boldsymbol{r}_{o}$, and hence requires 
the knowledge of the whole solvent velocity field $\boldsymbol{v}^{\text {solv }}\left(\boldsymbol{r}, t \leqslant t_{o}\right)$. However, making use of the short range of usual observables, we purport to bolster the intuition that, to leading order, $\left\langle g\left(\boldsymbol{r}_{0}\right)\right\rangle_{t_{o}}$ is mainly determined by the history of the velocity gradient $\boldsymbol{\kappa}\left(\boldsymbol{r}_{0}(t), t\right)$ along the solvent pathline $\boldsymbol{r}_{o}(t)$, with $\boldsymbol{r}\left(t_{o}\right)=\boldsymbol{r}_{o}$.

We define the range of a microscopic observable $g(\boldsymbol{r})$ as the distance beyond which the particle configuration becomes irrelevant. More precisely, range $(g)$ is the minimal radius of a disk $\mathcal{D}$ centred at $\boldsymbol{r}$ such that, for any two particle configurations $\Gamma^{(A)}$ and $\Gamma^{(B)}$ coinciding over $\mathcal{D}$, i.e., such that

$$
\boldsymbol{r}_{\boldsymbol{i}}^{(A)}=\boldsymbol{r}_{\boldsymbol{i}}^{(B)} \text { if } \boldsymbol{r}_{\boldsymbol{i}}^{(A)} \in \mathcal{D} \text { or } \boldsymbol{r}_{\boldsymbol{i}}^{(B)} \in \mathcal{D},
$$

$g\left(\boldsymbol{r}, \Gamma^{(A)}\right)$ and $g\left(\boldsymbol{r}, \Gamma^{(B)}\right)$ are equal, to a good approximation. For example, the range of the density observable $\rho(\boldsymbol{r}) \equiv \sum_{j=1}^{N} \delta\left(\boldsymbol{r}-\boldsymbol{r}_{\boldsymbol{j}}\right)$ is $0^{+}$and that of the stress $\boldsymbol{\sigma}(\boldsymbol{r})$ is bounded by the cut-off distance of interparticle interactions.

If the range of an observable $g\left(\boldsymbol{r}_{o}\right)$ is small compared to the lengthscale $l \sim \kappa / \nabla \kappa$ over which the velocity gradient varies, we are tempted to replace the global inhomogeneous flow with a much more tractable affine (i.e., homogeneous) velocity field that coincides with the inhomogeneous one around $\boldsymbol{r}_{o}$.

This comes down to approximating the genuine Smoluchowski operator (appearing, e.g., in Eq. 6) with

$$
\Omega_{\mathrm{hom}}^{\dagger}(\Gamma ; t)=\Omega_{\mathrm{eq}}^{\dagger}(\Gamma)+\sum_{i=1}^{N}\left[\boldsymbol{v}^{\mathrm{solv}}\left(\boldsymbol{r}_{o}, t\right)+\boldsymbol{\kappa}\left(\boldsymbol{r}_{o}, t\right) \cdot\left(\boldsymbol{r}_{\boldsymbol{i}}-\boldsymbol{r}_{o}\right)\right] \cdot \partial_{i},
$$

where

$$
\Omega_{\mathrm{eq}}^{\dagger}(\Gamma) \equiv \sum_{i=1}^{N}\left[\partial_{i}+\boldsymbol{F}_{\boldsymbol{i}}(\Gamma)\right] \cdot \partial_{i} .
$$

How large is the error due to this approximation? At time $t$, the error reads

$$
\left(e_{-}^{\int_{0}^{t} \Omega^{\dagger}(s) d s}-e_{-}^{\int_{0}^{t} \Omega_{\mathrm{hom}}^{\dagger}(s) d s}\right) g\left(\boldsymbol{r}_{o}, \Gamma\right) .
$$

In particular, at $t=0$, it is zero, and the first-order term in $t$ yields its initial growth rate,

$$
\begin{aligned}
\frac{1}{t} \int_{0}^{t}\left[\Omega^{\dagger}(s)-\Omega_{\mathrm{hom}}^{\dagger}(s)\right] g\left(\boldsymbol{r}_{o}, \Gamma\right) d s & =\mathcal{O}\left(\sum_{i=1}^{N}\|\nabla \boldsymbol{\kappa}\|\left\|\boldsymbol{r}_{\boldsymbol{i}}-\boldsymbol{r}_{o}\right\|^{2}\left|\partial_{i} g\left(\boldsymbol{r}_{o}, \Gamma\right)\right|\right) \\
& \left.=\mathcal{O}\left(N_{\mathcal{D}}(\Gamma)\|\nabla \boldsymbol{\kappa}\||\operatorname{range}(g)|^{2} \max _{i \in\{1, \ldots, N\}} \| \partial_{i} g\left(\boldsymbol{r}_{o}, \Gamma\right)\right) \|\right),
\end{aligned}
$$


where $N_{\mathcal{D}}(\Gamma)$ is the number of particles within disk $\mathcal{D}$ in configuration $\Gamma$. Clearly, $\|\nabla \boldsymbol{\kappa}\||\operatorname{range}(g)|^{2}$ arises because of the local deviations from affinity. The second-order term (quadratic in $\Omega^{\dagger}$ ) in the expansion of the approximation error also contains contributions in $\|\nabla \boldsymbol{\kappa}\||\operatorname{range}(g)|^{2}$; some are multiplied by $\Omega_{\text {eq }}^{\dagger}$ (which tends to restore the equilibrium configuration), while the others involve $\boldsymbol{v}^{\text {solv }}\left(\boldsymbol{r}_{o}, s\right)$, which drags particles away. Indeed, through advection with the solvent velocity, some particles, which initially lay far from $\boldsymbol{r}_{o}$ (where the affine approximation is poor), will enter the region $\mathcal{D}$ where they become relevant for the computation of $g$. Consequently, for the approximation to work best, particles close to $\boldsymbol{r}_{o}$ should move as little as possible. This is exactly why it is advantageous to switch to the auxiliary frame introduced in the previous section: the auxiliary driving field $\boldsymbol{v}^{\prime}\left(\boldsymbol{r}^{\prime}, t\right)$ vanishes at point $\boldsymbol{r}^{\prime}=\boldsymbol{r}_{o}$ at all times. In that frame, the approximate evolution is ruled by

$$
g^{\prime}\left(\boldsymbol{r}_{o}, \Gamma^{\prime} ; t_{0}\right)=e_{-}^{\int_{0}^{t_{0}} \Omega_{\mathrm{hom}}^{\dagger \prime}\left(\Gamma^{\prime} ; s\right) d s} g\left(\boldsymbol{r}_{o}, \Gamma^{\prime} ; 0\right),
$$

with

$$
\Omega_{\mathrm{hom}}^{\dagger \prime}\left(\Gamma^{\prime}, t\right) \equiv \sum_{i=1}^{N}\left[\partial_{i}^{\prime}+\boldsymbol{F}_{\boldsymbol{i}}{ }^{\prime}\left(\Gamma^{\prime}\right)+\boldsymbol{\kappa}^{\prime}\left(\boldsymbol{r}_{0}, t\right) \cdot\left(\boldsymbol{r}_{\boldsymbol{i}}{ }^{\prime}-\boldsymbol{r}_{0}\right)\right] \cdot \partial_{i}^{\prime} .
$$

Recalling that, by definition, the original frame and the auxiliary one coincide at time $t_{0}$ and thus $\left\langle g^{\prime}\left(\boldsymbol{r}_{o}\right)\right\rangle_{t_{o}}=\left\langle g\left(\boldsymbol{r}_{o}\right)\right\rangle_{t_{o}}$, we can now come back to the original frame, using the change of coordinates of Eq. 7, and confirm the intuition that, to leading order, $\left\langle g\left(\boldsymbol{r}_{o}\right)\right\rangle_{t_{o}}$ is governed by the velocity gradient along the pathline, i.e., $\left\{\boldsymbol{\kappa}\left(\boldsymbol{r}_{o}(t), t\right)\right\}$. This is the intrinsic part of the dynamics. As a reminder, the time derivative of $\left\langle g\left(\boldsymbol{r}_{o}\right)\right\rangle_{t_{o}}$ also involves an extrinsic part, namely, the advection term in Eq. 14

To proceed, physically motivated approximations, expressed as projections onto relevant variables, are performed onto the intrinsic dynamics. In the end, these approximations, conducted in Fourier space, shall heavily rely on the possibility to treat the driving flow as (almost) globally homogeneous, whereas the system under study may be globally very heterogeneous. The problem is solved by performing these approximations in the homogeneous auxiliary system of Eq. 17, which is a reasonable surrogate for the original one if the flow is locally homogeneous. This is a first step towards a

\footnotetext{
${ }^{1}$ Note, however, that the quality of this locally homogeneous approximation will dramatically worsen with the duration of the memory of the system and the magnitude of $\kappa$.
} 
systematic expansion of the velocity field in the auxiliary frame, starting (in this paper) with a uniform velocity gradient $\boldsymbol{\kappa}(\boldsymbol{r}, t)=\boldsymbol{\kappa}\left(\boldsymbol{r}_{0}(t), t\right)$, then considering the gradient $\boldsymbol{\nabla} \boldsymbol{\kappa}$ of $\boldsymbol{\kappa}$ at $\boldsymbol{r}_{0}(t)$, etc.

\section{Projection Scheme}

\subsection{Sets of slow variables}

In a typical mode-coupling spirit, the slow intrinsic evolution (with respect to microscopic time scales) of a generic observable $g$ such as the density or the stress will be captured via its projection onto (i.e., cross-correlation with) familiar slow modes. The observables will be expressed in Fourier space, where the collective dynamics are best captured. Since the global density $\rho_{\boldsymbol{q}=0}$ (where $\boldsymbol{q}$ represents a wavevector in Fourier space) is the only conserved quantity in the problem, i.e., $\partial_{t} \rho_{\boldsymbol{q}=0}=0$, and the relaxation time of $\rho_{\boldsymbol{q}}$ diverges in the limit of small $\boldsymbol{q}$, we define the linear density modes $\left\{\rho_{\boldsymbol{q}}, \boldsymbol{q} \in \mathbb{R}^{d}\right\}$ in Fourier space as a first set of slow modes, associated to the projector $P_{1}$, viz.,

$$
\left.P_{1} \equiv \sum_{\boldsymbol{q}} \rho_{\boldsymbol{q}}\right\rangle \frac{1}{N S_{\boldsymbol{q}}}\left\langle\rho_{\boldsymbol{q}}^{\star}\right.
$$

where $S_{\boldsymbol{q}} \equiv N^{-1}\left\langle\rho_{\boldsymbol{q}}^{\star} \rho_{\boldsymbol{q}}\right\rangle$ is the static structure factor, and its complementary part $Q_{1} \equiv 1-P_{1}$. It should be noted that the ensemble average in the projection is performed with respect to the equilibrium distribution $\psi_{\text {eq }}$ (denoted by $\langle\cdot\rangle$ here), whereas averages over the initial distribution $\psi_{0}$ shall be denoted by $\langle\cdot\rangle_{0}$,

$$
P_{1} g(\Gamma)=\frac{\rho_{\boldsymbol{q}}(\Gamma)}{N S_{q}}\left[\int \rho_{\boldsymbol{q}}^{\star}\left(\Gamma^{\prime}\right) g\left(\Gamma^{\prime}\right) \psi_{\mathrm{eq}}\left(\Gamma^{\prime}\right) d \Gamma^{\prime}\right] .
$$

In Ref. [12], Brader et al. noticed the absence of any coupling with linear density modes in a purely homogeneous flow and thus further projected the dynamics onto density pairs $\left\{\rho_{\boldsymbol{k}} \rho_{\boldsymbol{q}}, \boldsymbol{k}, \boldsymbol{q} \in \mathbb{R}^{d}\right\}$ with the projector

$$
\left.P_{2} \equiv \sum_{\boldsymbol{k}>\boldsymbol{q}} \rho_{\boldsymbol{k}} \rho_{\boldsymbol{q}}\right\rangle \frac{1}{N^{2} S_{\boldsymbol{k}} S_{\boldsymbol{q}}}\left\langle\rho_{\boldsymbol{k}}^{\star} \rho_{\boldsymbol{q}}^{\star},\right.
$$

where the Gaussian approximation $\left\langle\rho_{\boldsymbol{k}}^{\star} \rho_{\boldsymbol{q}}^{\star} \rho_{\boldsymbol{k}} \rho_{\boldsymbol{q}}\right\rangle \approx\left\langle\rho_{\boldsymbol{k}}^{\star} \rho_{\boldsymbol{k}}\right\rangle\left\langle\rho_{\boldsymbol{q}}^{\star} \rho_{\boldsymbol{q}}\right\rangle=N^{2} S_{\boldsymbol{k}} S_{\boldsymbol{q}}$ was used, and its complementary part $Q_{2}$. Although this section comes in the wake of Ref. [12], we shall not neglect the couplings with linear density modes from the outset, because the flow is not strictly homogeneous. 


\subsection{Generalised Green-Kubo relation}

To prepare the projection, we recast Eq. 6 into a form which better highlights the deviations from the initial configuration occurring throughout the past.

In the Schrödinger-like formulation, we denote by $\delta \psi$ these flow-induced perturbations, viz.,

$$
\psi(\Gamma, t)=\psi_{0}(\Gamma)+\delta \psi(\Gamma, t)
$$

Since $\partial_{t} \psi(\Gamma, t)=\Omega(\Gamma, t)\left[\psi_{0}(\Gamma)+\delta \psi(\Gamma, t)\right]$, solving for $\delta \psi$ yields

$$
\psi(\Gamma, t)=\psi_{0}(\Gamma)+\int_{0}^{t} d t_{1} e_{+}^{\int_{t_{1}}^{t} \Omega(\Gamma, s) d s} \Omega\left(\Gamma, t_{1}\right) \psi_{0}(\Gamma) .
$$

For the time being, $\Omega$ is the Smoluchowski operator for a generic flow field $\boldsymbol{v}^{\text {solv }}$, but the approximations performed in the following (see Section 4.1) will hinge on its being close to homogeneous, so one might already think of $\Omega$ and $\Omega^{\dagger}$ as their homogeneous auxiliary-frame surrogates of Eq. 17, that is to say, mentally consider the evolution in the auxiliary frame, with the replacement $\Omega^{\dagger} \rightarrow \Omega_{\mathrm{hom}}^{\dagger \prime}$.

Applying Eq. 18 to an arbitrary observable $g$, e.g., $g=\boldsymbol{\sigma}$, and partially integrating the time-ordered exponential, we arrive at a generalised GreenKubo (gGK) relation, expressed in the Heisenberg-like representation,

$$
\begin{aligned}
\langle g\rangle_{t} & =\int d \Gamma g(\Gamma)\left[\psi_{0}(\Gamma)+\int d \Gamma \int_{0}^{t} d t_{1} e_{+}^{\int_{t_{1}}^{t} \Omega(\Gamma, s) d s} \Omega\left(\Gamma, t_{1}\right) \psi_{0}(\Gamma)\right] \\
& =\langle g\rangle_{0}+\int_{0}^{t} d t_{1}\left\langle\Omega^{\dagger}\left(t_{1}\right) e_{-}^{\int_{t_{1}}^{t} \Omega^{\dagger}(s) d s} g\right\rangle_{0} .
\end{aligned}
$$

The Green-Kubo nature of Eq. 19 becomes clearer if the integrand is rewritten as

$$
\int d \Gamma\left[\Omega\left(\Gamma, t_{1}\right) \psi_{0}(\Gamma)\right] e_{-}^{\int_{t_{1}}^{t} \Omega^{\dagger}(s) d s} g
$$

$\Omega\left(\Gamma, t_{1}\right) \psi_{0}(\Gamma)$ is thus the deviation from $\psi_{0}(\Gamma)$ created at time $t_{1}$ (per unit time). For instance, for simple shear flow, starting with $\psi_{0}=\psi_{\text {eq }}$, the deviation couples strain rate and stress: $\Omega\left(\Gamma, t_{1}\right) \psi_{\text {eq }}(\Gamma)=\dot{\gamma}\left(t_{1}\right) \sigma_{x y}(\Gamma) \psi_{\text {eq }}(\Gamma)$, where $\sigma_{x y}$ is the shear element of the Kirkwood stress tensor and $\dot{\gamma}(t)$ is the imposed shear rate.

\subsection{Projected dynamics}

Let $U^{\dagger}\left(t, t_{1}\right)=e^{\int_{t_{1}}^{t} d t_{2} \Omega^{\dagger}\left(t_{2}\right)}$ be the propagator appearing in gGK (Eq. 19), associated with the full dynamics $\Omega^{\dagger}$. We split $U^{\dagger}$ into a part $U_{1}^{\dagger}\left(t, t_{1}\right) \equiv$ 


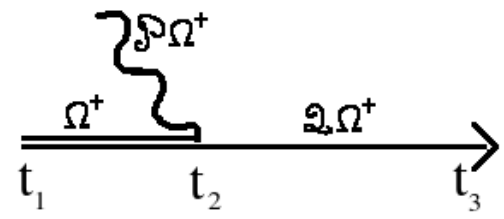

Figure 1: Schematic diagram of the decomposition of the full propagator $U^{\dagger}\left(t, t_{1}\right)$, associated with the operator $\Omega^{\dagger}$, as performed in Eq. 20 ,

$e^{\int_{t_{1}}^{t} d t_{1} Q_{1} \Omega^{\dagger}\left(t_{1}\right)}$ that evolves purely orthogonally to $P_{1}$ and a part that interacts at least once with $P_{1}$ (the notation $t_{2}$ referring to the time of the last interaction, see Fig. 10, viz.

$$
U^{\dagger}\left(t, t_{1}\right)=\int_{t_{1}}^{t} d t_{2} U^{\dagger}\left(t_{2}, t_{1}\right) P_{1} \Omega^{\dagger}\left(t_{2}\right) U_{1}^{\dagger}\left(t, t_{2}\right)+U_{1}^{\dagger}\left(t, t_{1}\right)
$$

Inserting the decomposition of Eq. 20 into gGK (Eq. 19), we arrive at:

$$
\begin{aligned}
\langle g\rangle_{t}-\langle g\rangle_{0}= & \int_{0}^{t} d t_{1}\left\langle\Omega^{\dagger}\left(t_{1}\right) U^{\dagger}\left(t, t_{1}\right) g\right\rangle_{0} \\
= & \int_{0}^{t} d t_{2} \int_{0}^{t_{1}} d t_{1}\left\langle\Omega^{\dagger}\left(t_{1}\right) U^{\dagger}\left(t_{2}, t_{1}\right) P_{1} \Omega^{\dagger}\left(t_{2}\right) U_{1}^{\dagger}\left(t, t_{2}\right) g\right\rangle_{0} \\
& +\int_{0}^{t} d t_{1}\left\langle\Omega^{\dagger}\left(t_{1}\right) U_{1}^{\dagger}\left(t, t_{1}\right) g\right\rangle_{0} \\
= & \int_{0}^{t} d t_{2} \sum_{\boldsymbol{q}} \underbrace{\int_{0}^{t_{2}} d t_{1}\left\langle\Omega^{\dagger}\left(t_{1}\right) U^{\dagger}\left(t_{2}, t_{1}\right) \rho_{\boldsymbol{q}}\right\rangle_{0}}_{\left\langle\rho_{\boldsymbol{q}}\right\rangle_{t_{2}}-\left\langle\rho_{\boldsymbol{q}}\right\rangle_{0}} \frac{\left\langle\rho_{\boldsymbol{q}}^{\star} \Omega^{\dagger}\left(t_{2}\right) U_{1}^{\dagger}\left(t, t_{2}\right) g\right\rangle}{N S_{\boldsymbol{q}}} \\
& +\underbrace{\int_{0}^{t} d t_{1}\left\langle\Omega^{\dagger}\left(t_{1}\right) U_{1}^{\dagger}\left(t, t_{1}\right) g\right\rangle_{0}}_{\left(\mathrm{Orth}_{1}\right)}
\end{aligned}
$$

where we have made use of gGK (applied to density fluctuations, $g \rightarrow \rho_{\boldsymbol{q}}$ ) in the last equality to reduce the first brace to $\left\langle\rho_{\boldsymbol{q}}\right\rangle_{t_{2}}-\left\langle\rho_{\boldsymbol{q}}\right\rangle_{0}$. One thus arrives at:

$$
\langle g\rangle_{t}-\langle g\rangle_{0}=\int_{0}^{t} d t_{2} \sum_{\boldsymbol{q}}\left(\left\langle\rho_{\boldsymbol{q}}\right\rangle_{t_{2}}-\left\langle\rho_{\boldsymbol{q}}\right\rangle_{0}\right) \frac{\left\langle\rho_{\boldsymbol{q}}^{\star} \Omega^{\dagger}\left(t_{2}\right) U_{1}^{\dagger}\left(t, t_{2}\right) g\right\rangle}{N S_{\boldsymbol{q}}}+\left(\mathrm{Orth}_{1}\right) .
$$


First, we focus on the dynamical correlator $\left\langle\rho_{\boldsymbol{q}}^{\star} \Omega^{\dagger}\left(t_{2}\right) U_{1}^{\dagger}\left(t, t_{2}\right) g\right\rangle$ on the rhs and introduce the identity $P_{1}+Q_{1}=1$ as follows:

$$
\begin{aligned}
\frac{\left\langle\rho_{\boldsymbol{q}}^{\star} \Omega^{\dagger}\left(t_{2}\right) U_{1}^{\dagger}\left(t, t_{2}\right) g\right\rangle}{N S_{\boldsymbol{q}}}= & \frac{1}{N S_{\boldsymbol{q}}}\left\langle\rho_{\boldsymbol{q}}^{\star} \Omega^{\dagger}\left(t_{2}\right) U_{1}^{\dagger}\left(t, t_{2}\right)\left(P_{1}+Q_{1}\right) g\right\rangle \\
= & -\sum_{\boldsymbol{k}} V_{\boldsymbol{k}}^{g} M_{\boldsymbol{q} \boldsymbol{k}}^{(1)}\left(t, t_{2}\right) \\
& +\frac{1}{N S_{\boldsymbol{q}}}\left\langle\rho_{\boldsymbol{q}}^{\star} \Omega^{\dagger}\left(t_{2}\right) U_{1}^{\dagger}\left(t, t_{2}\right) Q_{1} g\right\rangle,
\end{aligned}
$$

where the vertex $V_{\boldsymbol{k}}^{g} \equiv \frac{\left\langle\rho_{\boldsymbol{k}}^{\star} g\right\rangle}{N S_{\boldsymbol{k}}}$ quantifies the coupling of the observable $g$ to the density mode $\rho_{\boldsymbol{k}}$ in the equilibrium distribution and

$$
\begin{aligned}
M_{\boldsymbol{q} \boldsymbol{k}}^{(1)}\left(t, t_{2}\right) \equiv & -\frac{\left\langle\rho_{\boldsymbol{q}}^{\star} \Omega^{\dagger}\left(t_{2}\right) U_{1}^{\dagger}\left(t, t_{2}\right) \rho_{\boldsymbol{k}}\right\rangle}{N S_{\boldsymbol{q}}} \\
= & \frac{1}{N S_{\boldsymbol{q}}}\left\langle\left[\rho_{\boldsymbol{q}}^{\star} \sum_{j} \boldsymbol{v}^{\mathrm{solv}}\left(\boldsymbol{r}_{j}, t_{2}\right) \cdot \boldsymbol{F}_{j}\right.\right. \\
& \left.\left.+i \boldsymbol{q} \cdot\left(-\hat{\boldsymbol{F}}_{\boldsymbol{q}}{ }^{\star}+\hat{\boldsymbol{v}}_{\boldsymbol{q}}{ }^{\star}\left(t_{2}\right)\right)\right] U_{1}^{\dagger}\left(t, t_{2}\right) \rho_{\boldsymbol{k}}\right\rangle
\end{aligned}
$$

is a memory kernel evaluated in the equilibrium distribution, with

$$
\hat{\boldsymbol{F}}_{\boldsymbol{q}} \equiv \sum_{j} \boldsymbol{F}_{j} e^{-i \boldsymbol{q} \cdot \boldsymbol{r}_{j}} \text { and } \hat{\boldsymbol{v}}_{\boldsymbol{q}}(t) \equiv \sum_{j} \boldsymbol{v}^{\mathrm{solv}}\left(\boldsymbol{r}_{j}, t\right) e^{-i \boldsymbol{q} \cdot \boldsymbol{r}_{j}}
$$

\subsection{Application to the density observable}

Before turning to our main interest, i.e., the stress, we wish to illustrate the principle of the projection scheme for a generic flow, but on a simpler observable, namely, the density $g=\rho_{\boldsymbol{p}}, \boldsymbol{p} \in \mathbb{R}^{d}$, for which the complement $Q_{1} g$ vanishes by definition. The following calculations need not be performed in the homogeneous auxiliary frame; they hold true for an inhomogeneous flow.

Applying Eq. 22 to density modes $\left(V_{\boldsymbol{k}}^{\rho_{\boldsymbol{p}}} \equiv \frac{\left\langle\rho_{\boldsymbol{k}}^{\star} \rho_{\boldsymbol{p}}\right\rangle}{N S_{\boldsymbol{k}}}=\delta_{\boldsymbol{k}, \boldsymbol{p}}\right)$ leads to

$$
\left\langle\rho_{\boldsymbol{p}}\right\rangle_{t}-\left\langle\rho_{\boldsymbol{p}}\right\rangle_{0}=-\int_{0}^{t} d t_{1} \sum_{\boldsymbol{q}}\left[\left\langle\rho_{\boldsymbol{q}}\right\rangle_{t_{1}}-\left\langle\rho_{\boldsymbol{q}}\right\rangle_{0}\right] M_{\boldsymbol{q} \boldsymbol{p}}^{(1)}\left(t, t_{1}\right)+(\text { Orth } 1),
$$

where $M_{\boldsymbol{q} \boldsymbol{p}}^{(1)}\left(t, t_{1}\right)$ is given in Eq. 23 and $(\mathrm{Orth} 1) \equiv \int_{0}^{t} d t_{1}\left\langle\Omega^{\dagger}\left(t_{1}\right) U_{1}^{\dagger}\left(t, t_{1}\right) \rho_{\boldsymbol{p}}\right\rangle_{0}$. 
Taking a derivative with respect to time $t$ yields

$$
\begin{aligned}
\partial_{t}\left\langle\rho_{\boldsymbol{p}}\right\rangle_{t}= & -\sum_{\boldsymbol{q}}\left[\left\langle\rho_{\boldsymbol{q}}\right\rangle_{t}-\left\langle\rho_{\boldsymbol{q}}\right\rangle_{0}\right] M_{\boldsymbol{q} \boldsymbol{p}}^{(1)}(t, t) \\
& -\int_{0}^{t} d t_{1} \sum_{\boldsymbol{q}}\left[\left\langle\rho_{\boldsymbol{q}}\right\rangle_{t_{1}}-\left\langle\rho_{\boldsymbol{q}}\right\rangle_{0}\right] \partial_{t} M_{\boldsymbol{q} \boldsymbol{p}}^{(1)}\left(t, t_{1}\right)+\hat{S}_{\boldsymbol{p}}(t) .
\end{aligned}
$$

Here, we have used the explicit notation $\hat{S}_{\boldsymbol{p}}(t)$ for $\partial_{t}($ Orth1);

$\hat{S}_{\boldsymbol{p}}(t)=-i \boldsymbol{p} \cdot\left\langle\hat{\boldsymbol{F}}_{\boldsymbol{p}}+\hat{\boldsymbol{v}}_{\boldsymbol{p}}-i \boldsymbol{p} \rho_{\boldsymbol{p}}\right\rangle_{0}-\int_{0}^{t} d t_{1}\left\langle\Omega^{\dagger}\left(t_{1}\right) U_{1}^{\dagger}\left(t, t_{1}\right) Q_{1} i \boldsymbol{p} \cdot\left(\hat{\boldsymbol{F}}_{\boldsymbol{p}}+\hat{\boldsymbol{v}}_{\boldsymbol{p}}\right)\right\rangle_{0}$

The term $M_{\boldsymbol{q} \boldsymbol{p}}^{(1)}(t, t)=-\left(N S_{\boldsymbol{q}}\right)^{-1}\left\langle\rho_{\boldsymbol{q}}^{\star} \Omega^{\dagger}(t) \rho_{\boldsymbol{p}}\right\rangle$ can be simplified. Using the equilibrium (i.e., $\boldsymbol{v}^{\text {solv }}=\mathbf{0}$ ) Smoluchowski operator $\Omega_{\mathrm{eq}}^{\dagger}$ from Eq. 16, we can write

$$
\left\langle\rho_{\boldsymbol{q}}^{\star} \Omega^{\dagger}(t) \rho_{\boldsymbol{p}}\right\rangle=\left\langle\rho_{\boldsymbol{q}}^{\star} \Omega_{\mathrm{eq}}^{\dagger} \rho_{\boldsymbol{p}}\right\rangle+\left\langle\rho_{\boldsymbol{q}}^{\star} \sum_{j} \boldsymbol{v}^{\mathrm{solv}}\left(\boldsymbol{r}_{\boldsymbol{j}}, t\right) \cdot \partial_{j} \rho_{\boldsymbol{p}}\right\rangle,
$$

where, using partial integration,

$$
\begin{aligned}
\left\langle\rho_{\boldsymbol{q}}^{\star} \Omega_{\mathrm{eq}}^{\dagger} \rho_{\boldsymbol{p}}\right\rangle & =\left\langle\rho_{\boldsymbol{q}}^{\star} \sum_{j}\left(\partial_{j}+\boldsymbol{F}_{\boldsymbol{j}}\right) \cdot \partial_{j} \rho_{\boldsymbol{p}}\right\rangle \\
& =\int d \Gamma \psi_{\mathrm{eq}}(\Gamma) \rho_{\boldsymbol{q}}^{\star} \sum_{j}\left(\partial_{j}+\boldsymbol{F}_{\boldsymbol{j}}\right) \cdot \partial_{j} \rho_{\boldsymbol{p}} \\
& =-\int d \Gamma \sum_{j}\left\{\partial_{j}\left[\psi_{\mathrm{eq}}(\Gamma) \rho_{\boldsymbol{q}}^{\star}\right]-\rho_{\boldsymbol{q}}^{\star} \sum_{j} \boldsymbol{F}_{\boldsymbol{j}} \psi_{\mathrm{eq}}(\Gamma)\right\} \cdot \partial_{j} \rho_{\boldsymbol{p}} \\
& =-\boldsymbol{q} \cdot \boldsymbol{p}\left\langle\sum_{j} e^{i(\boldsymbol{q}-\boldsymbol{p}) \cdot \boldsymbol{r}_{j}}\right\rangle \\
& =-\boldsymbol{p}^{2} \delta_{\boldsymbol{q} \boldsymbol{p}} N
\end{aligned}
$$

The second term, $\left\langle\rho_{\boldsymbol{q}}^{\star} \sum_{j} \boldsymbol{v}^{\text {solv }}\left(\boldsymbol{r}_{\boldsymbol{j}}, t\right) \cdot \partial_{j} \rho_{\boldsymbol{p}}\right\rangle \equiv F_{\boldsymbol{q} \boldsymbol{p}}$, is most easily simplified 
by first backward-Fourier transforming it with respect to $\boldsymbol{p}$ only, viz.,

$$
\begin{aligned}
F_{\boldsymbol{q}}\left(\boldsymbol{r}_{0}\right) & =\left\langle\rho_{\boldsymbol{q}}^{\star} \sum_{j} \boldsymbol{v}^{\mathrm{solv}}\left(\boldsymbol{r}_{\boldsymbol{j}}, t\right) \cdot \partial_{j} \rho\left(\boldsymbol{r}_{0}\right)\right\rangle, \\
& =-\left\langle\rho_{\boldsymbol{q}}^{\star} \sum_{j} \boldsymbol{v}^{\mathrm{solv}}\left(\boldsymbol{r}_{\boldsymbol{j}}, t\right) \cdot \partial_{\boldsymbol{r}_{0}} \delta\left(\boldsymbol{r}_{0}-\boldsymbol{r}_{\boldsymbol{j}}\right)\right\rangle . \\
& =-\partial_{\boldsymbol{r}_{0}} \cdot\left\langle\rho_{\boldsymbol{q}}^{\star} \sum_{j} \boldsymbol{v}^{\mathrm{solv}}\left(\boldsymbol{r}_{\boldsymbol{j}}, t\right) \delta\left(\boldsymbol{r}_{0}-\boldsymbol{r}_{\boldsymbol{j}}\right)\right\rangle \\
& =-\left\langle\rho_{\boldsymbol{q}}^{\star} \boldsymbol{v}^{\mathrm{solv}}\left(\boldsymbol{r}_{0}, t\right) \cdot \partial_{\boldsymbol{r}_{0}} \rho\left(\boldsymbol{r}_{0}\right)\right\rangle,
\end{aligned}
$$

where we have used the incompressibility of the velocity field, viz., $\partial_{r_{0}}$. $\boldsymbol{v}^{\text {solv }}\left(\boldsymbol{r}_{0}, t\right)=0$. It suffices to transform $F_{\boldsymbol{q}}\left(\boldsymbol{r}_{0}\right)$ back into reciprocal space (with respect to $\boldsymbol{r}_{0}$ ) to obtain:

$$
\begin{aligned}
F_{\boldsymbol{q} \boldsymbol{p}} & =-\sum_{\boldsymbol{k}}\left\langle\rho_{\boldsymbol{q}}^{\star} \boldsymbol{v}_{\boldsymbol{k}}(t) \cdot i(\boldsymbol{p}-\boldsymbol{k}) \rho_{\boldsymbol{p}-\boldsymbol{k}}\right\rangle \\
& =-\boldsymbol{v}_{\boldsymbol{k}}(t) \cdot i \boldsymbol{q}\left\langle\rho_{\boldsymbol{q}}^{\star} \rho_{\boldsymbol{q}}\right\rangle \\
& =-i N \boldsymbol{v}_{\boldsymbol{p}-\boldsymbol{q}}^{\mathrm{solv}}(t) \cdot \boldsymbol{q} S_{\boldsymbol{q}} .
\end{aligned}
$$

Collecting these contributions into Eq. 25, one arrives at an equation of evolution of density fluctuations:

$$
\begin{gathered}
\partial_{t}\left\langle\rho_{\boldsymbol{p}}\right\rangle_{t}+\sum_{\boldsymbol{q}} \boldsymbol{v}_{\boldsymbol{p}-\boldsymbol{q}}^{\mathrm{solv}}(t) \cdot i \boldsymbol{q}\left\langle\rho_{\boldsymbol{q}}\right\rangle_{t}=-\frac{\boldsymbol{p}^{2}}{S_{\boldsymbol{p}}}\left\langle\rho_{\boldsymbol{p}}\right\rangle_{t}-\sum_{\boldsymbol{q}} \int_{0}^{t} d t_{1} \partial_{t} M_{\boldsymbol{q} \boldsymbol{p}}^{(1)}\left(t, t_{1}\right)\left\langle\rho_{\boldsymbol{q}}\right\rangle_{t_{1}} \\
+\hat{S}_{\boldsymbol{p}}(t) .
\end{gathered}
$$

Let us emphasise the physical meaning of the different terms:

(i) the second term on the lhs is the advection term established in Section 2.2 .

(ii) the first one on the rhs permits the relaxation of fluctuations through diffusion. Note that the normalising factor $S_{p}^{-1}$ is expected, because the relaxation of a density mode $\rho_{\boldsymbol{p}}$ does not require single-particle diffusion over a lengthscale $\|\boldsymbol{p}\|^{-1}$ ! Besides, if the density field is smoothed via coarsegraining (i.e., $\rho_{\boldsymbol{p}} \rightarrow \rho_{\boldsymbol{p}} \phi_{\boldsymbol{p}}$, with $\phi_{\boldsymbol{p}}$ akin to a Gaussian of half-width a few particle diameters), the fast relaxational modes at high $\boldsymbol{p}$ are suppressed, and the normalising factor then tends to $S_{\mathbf{0}}^{-1}$, which is directly related to the compressibility of the suspension. 
(iii) The second term on the rhs reflects the evolution in orthogonal space of the density fluctuations created in the past and their final coupling back to present fluctuations.

(iv) $\hat{S}_{\boldsymbol{p}}(t)$ is a source term that results from interactions with nonlinear density modes. Consistently with the expectation that density heterogeneities in an incompressible flow are due to collective effects, e.g., stress equilibration, it is the only term that can potentially create density inhomogeneities, insofar as the other terms are associated to pre-existing density heterogeneities. If a homogeneous flow is imposed to an initally uniform system, translational invariance imposes that, for finite $\boldsymbol{p},\left\langle\hat{\boldsymbol{F}}_{\boldsymbol{p}}\right\rangle_{0}=0,\left\langle\boldsymbol{\rho}_{\boldsymbol{p}}\right\rangle_{0}=0$, $i \boldsymbol{p} \cdot\left\langle\hat{\boldsymbol{v}}_{\boldsymbol{p}}\right\rangle_{0}=0$ in Eq. 26, ergo $\hat{S}_{\boldsymbol{p}}(t)=0$ at all times, for all $\boldsymbol{p}$; as expected, the source term then vanishes.

Finally, by comparing Eq. 27 to the mass conservation equation,

$$
\partial_{t}\left\langle\rho_{\boldsymbol{p}}\right\rangle_{t}+i \boldsymbol{p} \cdot\left\langle\boldsymbol{j}_{\boldsymbol{p}}^{\text {coll }}\right\rangle_{t}=0
$$

where $\boldsymbol{j}_{\boldsymbol{p}}^{\text {coll }} \equiv \sum_{j=1}^{N} \dot{\boldsymbol{r}}_{\boldsymbol{j}} e^{-i \boldsymbol{p} \cdot \boldsymbol{r}_{j}}$ is the colloidal flux, it can be seen that, in a heterogeneous flow, the colloidal velocity $\boldsymbol{u}^{\text {coll }}(\boldsymbol{r}, t) \equiv \frac{\left\langle\boldsymbol{j}^{\text {coll }}(\boldsymbol{r})\right\rangle_{t}}{\langle\rho(\boldsymbol{r})\rangle_{t}}$ will generally differ from the driving solvent velocity $\boldsymbol{v}^{\text {solv }}(\boldsymbol{r}, t)$.

\subsection{Orthogonal dynamics}

Let us come back to a generic observable $g$ and refine the description of term (Orth1) in Eq. 21. In principle, the propagator decomposition of Eq. 20 can be iterated, and the propagator $U_{1}^{\dagger}$, split into a part evolving with $P_{2} Q_{1} \Omega^{\dagger}$ and an orthogonal part $U_{2}^{\dagger}$, and so on, ad libitum. Schematically, one would then get

$$
\begin{aligned}
\langle g\rangle_{t} \approx & \langle g\rangle_{0}-\int_{0}^{t} d t_{2} \sum_{\boldsymbol{q}, \boldsymbol{k}}\left(\left\langle\rho_{\boldsymbol{q}}\right\rangle_{t_{2}}-\left\langle\rho_{\boldsymbol{q}}\right\rangle_{0}\right) V_{1}(\cdot) M_{\boldsymbol{q} \boldsymbol{k}}^{(1)}\left(t, t_{2}\right) \\
& -\int_{0}^{t} d t_{2} \sum_{\boldsymbol{k}, \boldsymbol{p}, \boldsymbol{k}^{\prime}, \boldsymbol{p}^{\prime}}\left(\left\langle\rho_{\boldsymbol{k}} \rho_{\boldsymbol{p}}\right\rangle_{t_{2}}-\left\langle\rho_{\boldsymbol{k}} \rho_{\boldsymbol{p}}\right\rangle_{0}\right) V_{2}(\cdot) M_{\boldsymbol{q} \boldsymbol{k} \boldsymbol{q}^{\prime} \boldsymbol{k}^{\prime}}^{(2)}\left(t, t_{2}\right)+\ldots
\end{aligned}
$$

and the orthogonal evolutions denoted by $M^{(n)}\left(t, t_{2}\right)$ would then be constrained to smaller and smaller spaces. However, following Ref. [12], we adopt a more pragmatic approach by directly introducing the projector $P_{2}$ 
in (Orth1), viz.,

$$
\begin{aligned}
\left(\mathrm{Orth}_{1}\right) & \equiv \int_{0}^{t} d t_{1}\left\langle\Omega^{\dagger}\left(t_{1}\right) Q_{1} U_{1}^{\dagger}\left(t, t_{1}\right) g\right\rangle_{0} \\
& \approx \int_{0}^{t} d t_{1}\left\langle\Omega^{\dagger}\left(t_{1}\right) Q_{1} P_{2} U_{1}^{\dagger}\left(t, t_{1}\right) P_{2} g\right\rangle_{0} \\
& \approx \int_{0}^{t} d t_{1} \sum_{\substack{\boldsymbol{k}>\boldsymbol{p} \\
\boldsymbol{k}^{\prime}>\boldsymbol{p}^{\prime}}} V_{0, \boldsymbol{k}, \boldsymbol{p}}\left(t_{1}\right) V_{\boldsymbol{k}^{\prime}, \boldsymbol{p}^{\prime}}^{g} \frac{\left\langle\rho_{\boldsymbol{k}}^{\star} \rho_{\boldsymbol{p}}^{\star} U_{1}^{\dagger}\left(t, t_{1}\right) \rho_{\boldsymbol{k}^{\prime}} \rho_{\boldsymbol{p}^{\prime}}\right\rangle}{N^{2} S_{\boldsymbol{k}} S_{\boldsymbol{p}}}
\end{aligned}
$$

where

$$
\begin{aligned}
V_{0, \boldsymbol{k}, \boldsymbol{p}}\left(t_{1}\right) & \equiv\left\langle\Omega^{\dagger}\left(t_{1}\right) Q_{1} \rho_{\boldsymbol{k}} \rho_{\boldsymbol{p}}\right\rangle_{0} \\
V_{\boldsymbol{k}^{\prime}, \boldsymbol{p}^{\prime}}^{g} & \equiv \frac{\left\langle\rho_{\boldsymbol{k}^{\prime}}^{*} \rho_{\boldsymbol{p}^{\prime}}^{*} g\right\rangle}{N^{2} S_{\boldsymbol{k}^{\prime}} S_{\boldsymbol{p}^{\prime}}}
\end{aligned}
$$

are vertices that represent, respectively, the creation and relaxation of bilinear density modes with respect to the initial configuration and the coupling strength of the observable $g$ to these modes. If the initial configuration $\psi_{0}$ and the velocity gradient at $t_{1}$ are close to homogeneous, then $V_{0, \boldsymbol{k}, \boldsymbol{p}}\left(t_{1}\right)$ is nonzero only for $\boldsymbol{p} \approx-\boldsymbol{k}$, and if $g$ is a spatial average, that is, if only smallwavenumber modes contribute to its Fourier decomposition, then $V_{\boldsymbol{k}^{\prime}, \boldsymbol{p}^{\prime}}^{g}$ is nonzero only if $\boldsymbol{p}^{\prime} \approx-\boldsymbol{k}^{\prime}$.

To conclude, the density-pair correlation function $\left\langle\rho_{\boldsymbol{k}}^{\star} \rho_{\boldsymbol{p}}^{\star} U_{1}^{\dagger}\left(t, t_{1}\right) \rho_{\boldsymbol{k}^{\prime}} \rho_{\boldsymbol{p}^{\prime}}\right\rangle$ is approximated through Gaussian factoring, which is a central approximation of mode-coupling theory [21]:

$$
\begin{aligned}
\frac{\left\langle\rho_{\boldsymbol{k}}^{\star} \rho_{\boldsymbol{p}}^{\star} U_{1}^{\dagger}\left(t, t_{1}\right) \rho_{\boldsymbol{k}^{\prime}} \rho_{\boldsymbol{p}^{\prime}}\right\rangle}{N^{2} S_{\boldsymbol{k}} S_{\boldsymbol{p}}} & \approx \frac{\left\langle\rho_{\boldsymbol{k}}^{\star} \rho_{\boldsymbol{p}}^{\star} U^{\dagger}\left(t, t_{1}\right) \rho_{\boldsymbol{k}^{\prime}} \rho_{\boldsymbol{p}^{\prime}}\right\rangle}{N^{2} S_{\boldsymbol{k}} S_{\boldsymbol{p}}} \\
& \approx \frac{\left\langle\rho_{\boldsymbol{k}}^{\star} U^{\dagger}\left(t, t_{1}\right) \rho_{\boldsymbol{k}^{\prime}}\right\rangle}{N S_{\boldsymbol{k}}} \frac{\left\langle\rho_{\boldsymbol{p}}^{\star} U^{\dagger}\left(t, t_{1}\right) \rho_{\boldsymbol{p}^{\prime}}\right\rangle}{N S_{\boldsymbol{p}}} \\
& =\Phi_{\boldsymbol{k} \boldsymbol{k}^{\prime}}\left(t, t_{1}\right) \Phi_{\boldsymbol{p} \boldsymbol{p}^{\prime}}\left(t, t_{1}\right),
\end{aligned}
$$

where we have introduced the transient density correlator

$$
\Phi_{\boldsymbol{q} \boldsymbol{k}}\left(t, t_{2}\right) \equiv \frac{\left\langle\rho_{\boldsymbol{q}}^{\star} U^{\dagger}\left(t, t_{2}\right) \rho_{\boldsymbol{k}}\right\rangle}{N S_{\boldsymbol{q}}}
$$


This correlator indicates how fast density fluctuations relax, in the presence of flow. A central result of the rheological extension of MCT by Brader and colleagues [12] ascertains that, for a translationally invariant (i.e., homogeneous) flow, $\Phi_{\boldsymbol{q} \boldsymbol{k}}\left(t, t_{2}\right)$ is non-zero only if $\boldsymbol{q}$ coincides with $\boldsymbol{k}$ in the solvent flow frame, i.e., if the flow advects wavevector $\boldsymbol{k}$ at time $t_{2}$ into $\boldsymbol{q}=\boldsymbol{k}\left(t, t_{2}\right) \equiv e_{+}^{\int_{t_{2}}^{t} d s \boldsymbol{\kappa}(s)}$ at a later time $t$, where $\boldsymbol{\kappa}$ is the uniform velocity gradient (recall $\left.\kappa_{\alpha \beta}(\boldsymbol{r}, t) \equiv \partial_{\beta} v_{\alpha}^{\text {solv }}(\boldsymbol{r}, t)\right)$. Interestingly, the norm of $\boldsymbol{k}\left(t, t_{2}\right)$ increases with the deformation; consequently, $\Phi$ is effectively evaluated at a smaller lengthscale, where thermal relaxation occurs faster. But flow heterogeneities induce additional cross-couplings between $\rho_{\boldsymbol{q}}^{\star}$ and $U^{\dagger}\left(t, t_{2}\right) \rho_{\boldsymbol{k}}$, through the interaction between the structure (the density modes $\rho_{\boldsymbol{q}}$ and $\rho_{\boldsymbol{k}}$ ) and the flow field (the velocity gradient modes $\boldsymbol{\kappa}_{\boldsymbol{p}}(s)$ )

After collecting all terms, we arrive at the following expression for Eq. 21.

$$
\begin{aligned}
& \langle g\rangle_{t}-\langle g\rangle_{0} \approx-\int_{0}^{t} d t_{1} \sum_{\boldsymbol{q}}\left(\left\langle\rho_{\boldsymbol{q}}\right\rangle_{t_{1}}-\left\langle\rho_{\boldsymbol{q}}\right\rangle_{0}\right) \\
& \times\left[\sum_{\boldsymbol{k}} V_{\boldsymbol{k}}^{g} M_{\boldsymbol{q} \boldsymbol{k}}^{(1)}\left(t, t_{1}\right)+\frac{1}{N S_{\boldsymbol{q}}}\left\langle\rho_{\boldsymbol{q}}^{\star} \Omega^{\dagger}\left(t_{1}\right) U_{1}^{\dagger}\left(t, t_{1}\right) Q_{1} g\right\rangle\right] \\
& +\int_{0}^{t} d t_{1} \sum_{\substack{\boldsymbol{k}>\boldsymbol{p} \\
\boldsymbol{k}^{\prime}>\boldsymbol{p}^{\prime}}} V_{0, \boldsymbol{k}, \boldsymbol{p}}\left(t_{1}\right) V_{\boldsymbol{k}^{\prime}, \boldsymbol{p}^{\prime}}^{g} \Phi_{\boldsymbol{k} \boldsymbol{k}^{\prime}}\left(t, t_{1}\right) \Phi_{\boldsymbol{p \boldsymbol { p } ^ { \prime }}}\left(t, t_{1}\right) .
\end{aligned}
$$

We should pay attention to a possible issue with Eq. 30: if one is not cautious, there is a risk that the uncontrolled approximation of (Orth1) may create spurious inhomogeneities that violate translational invariance, even in cases where it should theoretically be obeyed. We shall ward off this risk in a pragmatic way by making a judicious choice for the transient density correlator and ensuring the respect of fundamental physical principles in the final equations.

\footnotetext{
${ }^{2}$ This can be seen by replacing $U^{\dagger}\left(t, t_{2}\right) \rho_{\boldsymbol{k}}$ in $\Phi_{\boldsymbol{q} \boldsymbol{k}}\left(t, t_{2}\right)$ with its gGK expression (Eq. 19). The resulting integral involves a rate of deviation $\Omega(s) \psi_{\text {eq }}=-\sum_{j} \boldsymbol{v}^{\text {solv }}\left(\boldsymbol{r}_{j}, s\right)$. $\boldsymbol{F}_{\boldsymbol{j}} \psi_{\text {eq }}$, which is proportional to $\boldsymbol{\kappa}_{\mathbf{0}}(s): \boldsymbol{\sigma}_{\mathbf{0}}$ if the flow is homogeneous, but comprises non-zero Fourier modes otherwise.
} 


\section{Severe approximations lead to a constitutive equation of the White-Metzner class}

Armed with the projection scheme of the previous part, we are now capable of studying the stress observable $\boldsymbol{\sigma}_{\boldsymbol{q}}$. Direct application of Eq. 30 gives

$$
\begin{aligned}
\left\langle\boldsymbol{\sigma}_{\boldsymbol{q}}\right\rangle_{t}-\left\langle\boldsymbol{\sigma}_{\boldsymbol{q}}\right\rangle_{0} \approx & -\int_{0}^{t} d t_{1} \sum_{\boldsymbol{q}}\left(\left\langle\rho_{\boldsymbol{q}}\right\rangle_{t_{1}}-\left\langle\rho_{\boldsymbol{q}}\right\rangle_{0}\right) \\
& \times\left[\sum_{\boldsymbol{k}} V_{\boldsymbol{k}}^{\boldsymbol{\sigma}_{\boldsymbol{q}}} M_{\boldsymbol{q} \boldsymbol{k}}^{(1)}\left(t, t_{1}\right)+\frac{1}{N S_{\boldsymbol{q}}}\left\langle\rho_{\boldsymbol{q}}^{\star} \Omega^{\dagger}\left(t_{1}\right) U_{1}^{\dagger}\left(t, t_{1}\right) Q_{1} \boldsymbol{\sigma}_{\boldsymbol{q}}\right\rangle\right] \\
& +\int_{0}^{t} d t_{1} \sum_{\substack{\boldsymbol{k}>\boldsymbol{p} \\
\boldsymbol{k}^{\prime}>\boldsymbol{p}^{\prime}}} V_{0, \boldsymbol{k}, \boldsymbol{p}}\left(t_{1}\right) V_{\boldsymbol{k}^{\prime}, \boldsymbol{p}^{\prime}}^{\boldsymbol{\sigma}_{\boldsymbol{q}}} \Phi_{\boldsymbol{k} \boldsymbol{k}^{\prime}}\left(t, t_{1}\right) \Phi_{\boldsymbol{p} \boldsymbol{p}^{\prime}}\left(t, t_{1}\right) .
\end{aligned}
$$

The usual MCT protocol involves the derivation of equations for the transient density correlators $\Phi_{\boldsymbol{q} p}\left(t, t_{1}\right)$, with the help of the Zwanzig-Mori projection formalism. However, in the presence of flow heterogeneities and in non-trivial geometry, this would be a difficult task, which we bypass here: bearing in mind our main goal, namely, a study of the visco-elastic instability, we opt for a drastic simplification of the equations. We shall thus perform very strong, and mostly uncontrolled (but explicitly mentioned) approximations and we do not expect them to preserve the quantitative details of the full theory. Nevertheless, we aim to arrive at a tractable model that displays shear-thinning and correctly captures the low-shear-rate and highshear-rate regime of the flow. This will allow us to test the phenomenology of our extension of the framework to inhomogeneous situations and flow instabilities.

\subsection{Reduction to a locally homogeneous flow}

To start with, we assume that the flow only moderately deviates from homogeneity, so that the locally homogeneous flow approximation of Section 2.3 is valid. Under that assumption, the source term $\hat{S}_{p}(t)$ for the density in Eq. 27 vanishes, and no density inhomogeneity is created (effects such as shear-concentration coupling [22, 23] are thereby precluded). Thus, we suppose $\left\langle\rho_{\boldsymbol{q}}\right\rangle_{t} \approx\left\langle\rho_{\boldsymbol{q}}\right\rangle_{0} \approx N \delta_{\boldsymbol{q}, \mathbf{0}}$.

Furthermore, if the velocity gradient varies little over the distance travelled by a material volume before its microstructure relaxes, the intrinsic 
dynamics of the local stress $\boldsymbol{\sigma}(\boldsymbol{r}, t)$ are governed by the history of the lo$c a l$ velocity gradient $\left\{\boldsymbol{\kappa}\left(\boldsymbol{r}, t^{\prime}\right), t^{\prime}<t\right\}$. In other words, all non-local effects due to, e.g., stress waves emitted by distant regions are discarded. Within mesoscopic regions of the material, translational invariance is obeyed, so that only one cross-coupling subsists in the transient density correlator, viz., $\Phi_{\boldsymbol{k} \boldsymbol{k}^{\prime}}\left(t, t_{1}\right)=\Phi_{\boldsymbol{k} \boldsymbol{k}^{\prime}}\left(t, t_{1}\right) \delta_{\boldsymbol{k}, \boldsymbol{k}^{\prime}\left(t, t_{1}\right)}$. Under these assumptions, the vertices in Eq. 31 boil down to [12]

$$
\begin{aligned}
& V_{\boldsymbol{k}^{\prime}, \boldsymbol{p}^{\prime}}^{\sigma}=\boldsymbol{k}^{\prime} \boldsymbol{p}^{\prime} \frac{S_{\boldsymbol{k}^{\prime}}^{\prime}}{N^{2} k^{\prime} S_{\boldsymbol{k}^{\prime}} S_{\boldsymbol{p}^{\prime}}} \delta_{\boldsymbol{k}^{\prime},-\boldsymbol{p}^{\prime}}, \\
& V_{0, \boldsymbol{k}, \boldsymbol{p}}\left(t_{1}\right)=\boldsymbol{\kappa}\left(t_{1}\right): \boldsymbol{k} \boldsymbol{p} \frac{S_{\boldsymbol{k}}^{\prime}}{k} \delta_{\boldsymbol{k},-\boldsymbol{p}} .
\end{aligned}
$$

Thus, we recover the formula for homogeneous flow derived by Brader et al. [15, 12], namely,

$\boldsymbol{\sigma}(t)=-\int_{0}^{t} d t_{1} \sum_{\boldsymbol{k}^{\prime}} A\left(\boldsymbol{k}^{\prime}, t, t_{1}\right)\left[\frac{\partial}{\partial t_{1}}\left(\boldsymbol{k}^{\prime} \cdot \boldsymbol{B}\left(t, t_{1}\right) \cdot \boldsymbol{k}^{\prime}\right)\right] \Phi_{\boldsymbol{k}^{\prime}\left(t, t_{1}\right)}^{2}\left(t, t_{1}\right) \boldsymbol{k}^{\prime} \otimes \boldsymbol{k}^{\prime}$,

where where we have introduced the Finger tensor $\boldsymbol{B}\left(t, t_{1}\right) \equiv e_{+}^{\int_{t_{1}}^{t} d s \boldsymbol{\kappa}(s)}$. $e_{-}^{\int_{t_{1}}^{t} d s \boldsymbol{\kappa}^{\top}(s)}$ and $A\left(\boldsymbol{k}^{\prime}, t, t_{1}\right) \propto \frac{S_{\boldsymbol{k}^{\prime}}^{\prime} S_{\boldsymbol{k}^{\prime}\left(t, t_{1}\right)}^{\prime}}{k^{\prime} k^{\prime}\left(t, t_{1}\right) S_{\boldsymbol{k}^{\prime}}^{2}}$ collects all relevant equilibrium properties of the material.

\subsection{Schematic approximation}

To proceed, we follow the schematic approximation conducted in Ref. [15] by dropping all existing wavevector dependences (or, better said, by focusing on the most relevant wavevector) in Eq. 32, viz.,

$$
\boldsymbol{\sigma}(t)=-v_{\sigma} \int_{0}^{t} d t_{1} \frac{\partial \boldsymbol{B}\left(t, t_{1}\right)}{\partial t_{1}} \Phi^{2}\left(t, t_{1}\right)
$$

where $v_{\sigma} \approx k_{B} T n$ (with $n$ the number density) sets the scale of stress fluctuations. Partial integration yields

$$
\boldsymbol{\sigma}(t)=v_{\sigma} \int_{0}^{t} d t_{1} \boldsymbol{B}\left(t, t_{1}\right) \frac{\partial \Phi^{2}\left(t, t_{1}\right)}{\partial t_{1}}
$$




\subsection{Approximation of the transient density correlator}

At rest, the thermal relaxation of density fluctuations takes a time $\tau_{\alpha}$ that, according to MCT, diverges in the ideal glassy phase. But the presence of a solvent flow distorts the material structure and accelerates this relaxation. Arguably our crudest assumption will now consist in proposing a "phenomenological" characteristic time scale $\tau$ for the long-run decay of the transient density correlator, which takes this flow-induced relaxation into account:

$$
\begin{aligned}
\frac{\partial}{\partial t} \Phi\left(t, t_{1}\right) & =-\frac{\Phi\left(t, t_{1}\right)}{2 \tau[\dot{\boldsymbol{\epsilon}}(t)]} \\
\frac{\partial}{\partial t_{1}} \Phi\left(t, t_{1}\right) & =\frac{\Phi\left(t, t_{1}\right)}{2 \tau\left[\dot{\boldsymbol{\epsilon}}\left(t_{1}\right)\right]} \\
\text { with } \tau[\dot{\boldsymbol{\epsilon}}] & \equiv \frac{\tau_{\alpha}}{1+2 \alpha \tau_{\alpha} \sqrt{J_{2}(\dot{\boldsymbol{\epsilon}})}}
\end{aligned}
$$

Here, $\dot{\boldsymbol{\epsilon}}(t) \equiv \frac{\boldsymbol{\kappa}(t)+\boldsymbol{\kappa}^{\top}(t)}{2}$ is the strain rate tensor, $J_{2}$ is the second tensorial invariant of a deviatoric tensor, viz., $J_{2}(\dot{\boldsymbol{\epsilon}})=\frac{1}{2} \dot{\epsilon}_{i j} \dot{\epsilon}_{j i}$ (this reduces to $J_{2}(\boldsymbol{\kappa})=$ $\frac{1}{4} \dot{\gamma}^{2}$ under simple shear), and $\alpha$ is a material parameter that quantifies shearthinning. More precisely, $\alpha^{-1}$ is an "inverse yield strain" that describes how much strain is required to erase the memory of the local structure [15]. The following limit cases are enlightening: at vanishing shear rate, $\tau(\dot{\boldsymbol{\epsilon}})$ tends to the quiescent relaxation time $\tau_{\alpha}$, while at high shear rates one gets $\tau(\dot{\boldsymbol{\epsilon}}) \approx(\alpha \dot{\gamma})^{-1}$ (under simple shear).

Equations $[35,36$ lead to

$$
\Phi^{2}\left(t, t_{1}\right)=\exp \left(-\int_{t_{1}}^{t} \frac{d s}{\tau[\dot{\boldsymbol{\epsilon}}(s)]}\right) .
$$

\subsection{Constitutive equation}

With these severe approximations in hand, we now differentiate Eq. 34 with respect to time $t$.

$$
\frac{\partial}{\partial t} \boldsymbol{\sigma}(t)=\frac{v_{\sigma}}{\tau[\dot{\boldsymbol{\epsilon}}(t)]} \mathbb{I}+\boldsymbol{\kappa}(t) \cdot \boldsymbol{\sigma}(t)+\boldsymbol{\sigma}(t) \cdot \boldsymbol{\kappa}(t)^{\top}-\frac{\boldsymbol{\sigma}(t)}{\tau[\dot{\boldsymbol{\epsilon}}(t)]},
$$

where $\mathbb{I}$ is the identity matrix and we have used the equality $\left.\frac{\partial \Phi^{2}\left(t, t_{1}\right)}{\partial t_{1}}\right|_{t_{1}=t}=$ $\tau[\dot{\boldsymbol{\epsilon}}(t)]^{-1}$ as well as $\frac{\partial \boldsymbol{B}\left(t, t_{1}\right)}{\partial t}=\boldsymbol{\kappa}(t) \cdot \boldsymbol{B}\left(t, t_{1}\right)+\boldsymbol{B}\left(t, t_{1}\right) \cdot \boldsymbol{\kappa}(t)^{\top}$. Splitting 
$\boldsymbol{\sigma}$ into a quiescent $\left(\right.$ i.e., $\boldsymbol{\kappa}=\mathbf{0}$ ) pressure part $v_{\sigma} \mathbb{I}$ and a driven part $\boldsymbol{\sigma}^{\mathrm{d}}$ in Eq. 39, we arrive at

$$
\frac{\partial}{\partial t} \boldsymbol{\sigma}^{\mathrm{d}}(t)=v_{\sigma}\left[\boldsymbol{\kappa}(t)+\boldsymbol{\kappa}^{\top}(t)\right]+\boldsymbol{\kappa}(t) \cdot \boldsymbol{\sigma}^{\mathrm{d}}(t)+\boldsymbol{\sigma}^{\mathrm{d}}(t) \cdot \boldsymbol{\kappa}(t)^{\top}-\frac{\boldsymbol{\sigma}^{\mathrm{d}}(t)}{\tau[\dot{\boldsymbol{\epsilon}}(t)]} .
$$

Finally, one should recall that Eq. 40 deals with the intrinsic dynamics, i.e., the evolution in the (homogeneous) auxiliary frame introduced in Eq. 17. The full evolution of the observable in the lab frame is recovered by applying Eq. 14, which restores the inhomogeneity advection term $\boldsymbol{v} \cdot \nabla \boldsymbol{\sigma}$ found in Section 2.2, responsible for the advection of stress fluctuation with the microstructure, hence with the flow. After dropping the "d" superscripts, we thus obtain

$$
\frac{\mathfrak{D}}{\mathfrak{D} t} \boldsymbol{\sigma}(\boldsymbol{r}, t)+\frac{\boldsymbol{\sigma}(\boldsymbol{r}, t)}{\tau[\dot{\boldsymbol{\epsilon}}(\boldsymbol{r}, t)]}=2 v_{\sigma} \dot{\boldsymbol{\epsilon}}(\boldsymbol{r}, t),
$$

where the upper convected derivative (a.k.a. advected Truesdell derivative) is defined by

$$
\frac{\mathfrak{D} \boldsymbol{\sigma}}{\mathfrak{D} t} \equiv \frac{\partial \boldsymbol{\sigma}}{\partial t}+\boldsymbol{v} \cdot \nabla \boldsymbol{\sigma}-\boldsymbol{\kappa}(t) \cdot \boldsymbol{\sigma}(t)-\boldsymbol{\sigma}(t) \cdot \boldsymbol{\kappa}(t)^{\top} .
$$

Remember that the final relaxation time depends on the intrinsic dynamics and the shear rate and is given in Eq. 37

Interestingly, this relatively simple constitutive equation belongs to a class of (mostly) phenomenological models initially put forward in the context of polymer melt rheology by White and Metzner (WM) [16], on the basis of symmetry considerations. It obeys the principles of locality, causality and material objectivity.

In addition to the configuration-based stress $\boldsymbol{\sigma}$, which is associated to the colloidal microstructure, we include a Newtonian contribution to the stress accounting for viscous dissipation; the latter can indeed become significant at large shear rates. The total stress then reads

$$
\boldsymbol{\Sigma}=\boldsymbol{\sigma}+2 \eta_{s} \dot{\boldsymbol{\epsilon}}
$$

Note that this model has already been studied by Papenkort and Voigtmann in the case of a channel flow [24], where however the advected derivative played no role. 


\subsection{Bulk rheology and parameter fitting}

Within the WM-type model, we first consider the rheological properties of the bulk shear flow, i.e., $\boldsymbol{\kappa}=\dot{\gamma}^{\star} \boldsymbol{e}_{\mathbf{1}} \otimes \boldsymbol{e}_{\mathbf{2}}$, prior to any potential instability. From the constitutive equation, using $\eta_{0}$ as a shorthand for $v_{\sigma} \tau_{\alpha}$, it is easy to show that

$$
\begin{aligned}
\sigma_{11}^{\star} & =0 \\
\sigma_{12}^{\star} & =\frac{\eta_{0} \dot{\gamma}^{\star}}{1+\alpha \tau_{\alpha}\left|\dot{\gamma}^{\star}\right|} \\
\sigma_{22}^{\star} & =2 \eta_{0} \tau_{\alpha}\left(\frac{\dot{\gamma}^{\star}}{1+\alpha \tau_{\alpha}\left|\dot{\gamma}^{\star}\right|}\right)^{2} .
\end{aligned}
$$

Turning to linear rheology, the storage and loss moduli associated to the WM-type model are identical to those of a Maxwell model with a Newtonian contribution, viz.,

$$
\begin{aligned}
G^{\prime}(\omega) & =\frac{\eta_{0} \tau_{\alpha} \omega^{2}}{1+\omega^{2} \tau_{\alpha}^{2}} \\
G^{\prime \prime}(\omega) & =\frac{\eta_{0} \omega}{1+\omega^{2} \tau_{\alpha}^{2}}+\eta_{s} \omega .
\end{aligned}
$$

To fit the model parameters $v_{\sigma}, \tau_{\alpha}, \alpha$, and $\eta_{s}$, we compare the $\sigma_{12}^{\star}\left(\dot{\gamma}^{\star}\right)$ flow curve and, with less emphasis, the storage and loss moduli to experimental measurements by Siebenbürger et al. on suspensions of $\sim 100 \mathrm{~nm}$-large colloids with a thermosensitive PNIPAM shell (which affords a sensitive control of the effective volume fraction through the tuning of the temperature) [25]. In Ref. [25], a schematic version of the MCT equations was shown to provide excellent agreement with both the oscillatory shear and the steady shear measurements, for several effective volume fractions across the glass transition, while the solutions of the microscopic MCT equations were tested in Ref. [14] and yielded a consistent first principles description.

Figure 2 presents the flow curve fits obtained with our much cruder (but also much more tractable) constitutive equation; there is no doubt that the quality of the fits has suffered from our strong approximations: this confirms that the memory effects encoded in $\Phi\left(t, t_{1}\right)$ are more subtle than our simple Ansatz of Eq. 37. Nevertheless, the downward bending of the stress at low shear rates, which originates from thermal relaxation, and the strong shear-thinning effects, as well as the viscous behaviour at high shear rates are reasonably well captured. Let us also mention that our fitted model parameters (see Table 1) have values comparable to the corresponding quantities 


\begin{tabular}{|c|c|c|c|c|}
\hline$\phi_{\text {eff }}$ & $\tau_{\alpha}$ & $\alpha$ & $\eta_{0}$ & $\eta_{s}$ \\
\hline \hline 0.519 & 1.7 & 22 & 9.0 & 1.2 \\
\hline 0.600 & 900 & 35 & $8.1 \cdot 10^{3}$ & 1.3 \\
\hline 0.626 & $6.0 \cdot 10^{4}$ & 30 & $5.4 \cdot 10^{5}$ & 1.5 \\
\hline 0.639 & $8.0 \cdot 10^{5}$ & 30 & $6.4 \cdot 10^{6}$ & 1.9 \\
\hline 0.641 & $3.0 \cdot 10^{6}$ & 40 & $4.8 \cdot 10^{7}$ & 2.0 \\
\hline
\end{tabular}

Table 1: Model parameters used to fit the experimental flow curves in Fig. 2 and linear spectra in Fig. 3 .

As in Fig. 2, stresses are in units of $\frac{R_{h}^{3}}{k_{B} T}$ and times in units of $\frac{k_{B} T}{6 \pi \eta_{\mathrm{solv}} R_{h}^{3}}$.

in Ref. [25]; note, for instance, that $\tau_{\alpha}$ increases dramatically with $\phi_{\text {eff }}$ and that $\alpha$ is roughly of the order of the inverse yield strain.

We bestowed less importance to the linear rheology moduli $G^{\prime}$ and $G^{\prime \prime}$ in fitting the model parameters, because the rest of the paper deals with steady shear; still, Fig. 3 shows that the experimentally observed trends are also present in the model, although there is no quantitative agreement. The origin of the deviations is the broad distribution of relaxation times neglected in the White Metzner model but contained in the MCT description.

\subsection{Base flow in Taylor-Couette geometry}

Now, we focus on the base flow in a curved geometry, and more specifically in the Taylor-Couette cell used by Siebenbürger and colleagues in Ref. [25]. In such a rheometer, the fluid flows in the annular region between two co-axial cylinders, of radii $r_{i}=13.33 \mathrm{~mm}$ and $r_{o}=14.46 \mathrm{~mm}$ (relative gap width $\epsilon=0.085$ ), due to the rotation of the inner one. This geometry will be kept fixed for the rest of the paper.

Turning to the determination of the flow, we note that, unlike in Section 2, the velocity profile $\boldsymbol{v}(\boldsymbol{r})$ is no longer prescribed. Therefore, we need to close the equations by complementing the WM-type constitutive equation, (Eq. 41) with the inertialess momentum conservation equation,

$$
0=\nabla \cdot \boldsymbol{\Sigma}-\nabla p
$$




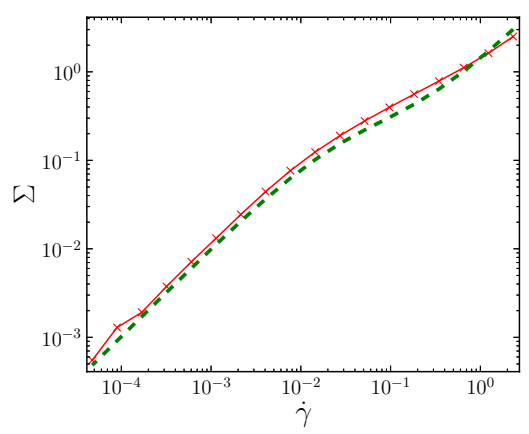

(a) $\phi_{\text {eff }}=0.519$

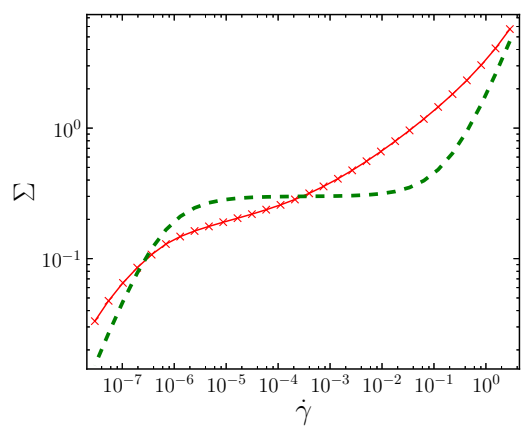

(c) $\phi_{\text {eff }}=0.626$

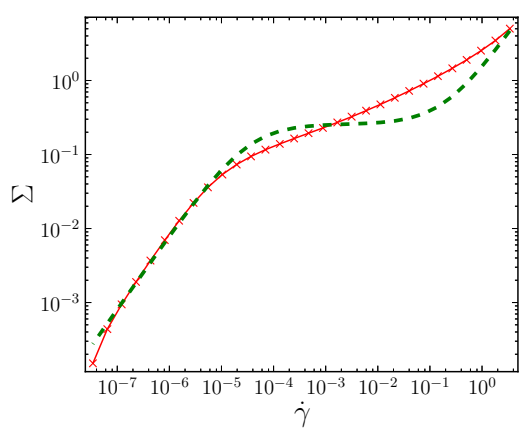

(b) $\phi_{\text {eff }}=0.600$

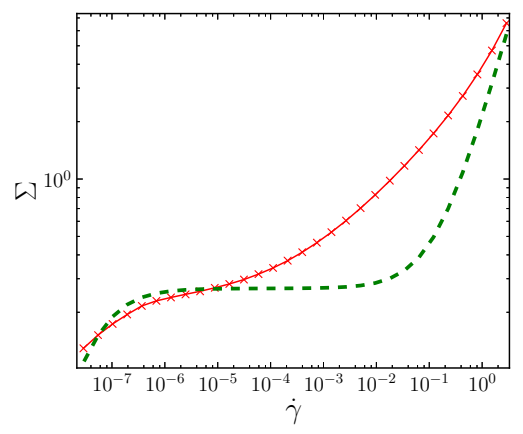

(d) $\phi_{\text {eff }}=0.639$

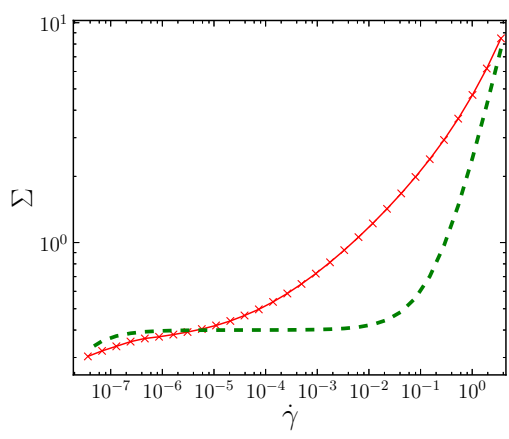

(e) $\phi_{\text {eff }}=0.641$

Figure 2: Flow curves for different effective volume fractions. (Red lines with crosses) experimental measurements from Ref. [25]; (dashed green lines) fits with the WM-type model (see text); parameters are listed in Table I.

Stresses are given in units of $\frac{R_{h}^{3}}{k_{B} T}$, where $R_{h}$ is the hydrodynamic radius of the colloids, and shear rates are non-dimensionalised with $\frac{6 \pi \eta_{\text {solv }} R_{h}^{3}}{k_{B} T}$, with $\eta_{\text {solv }}$ the solvent viscosity. 


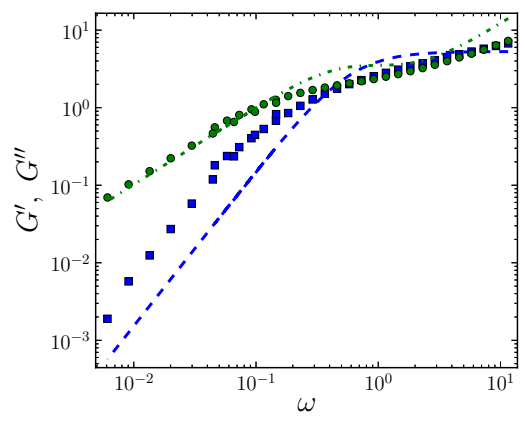

(a) $\phi_{\text {eff }}=0.519$

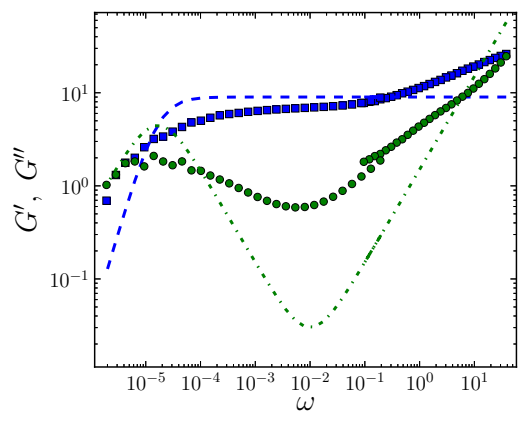

(c) $\phi_{\text {eff }}=0.626$

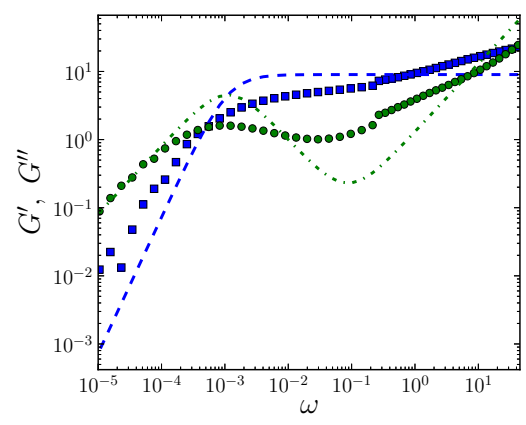

(b) $\phi_{\text {eff }}=0.600$

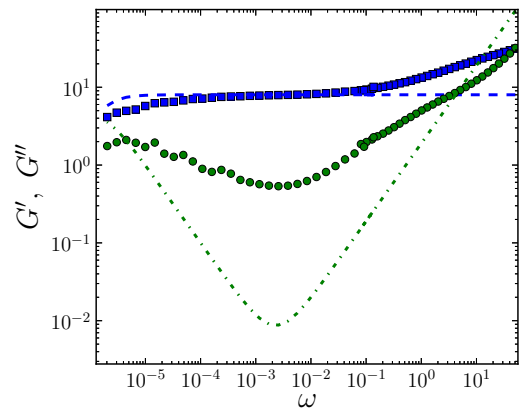

(d) $\phi_{\text {eff }}=0.639$

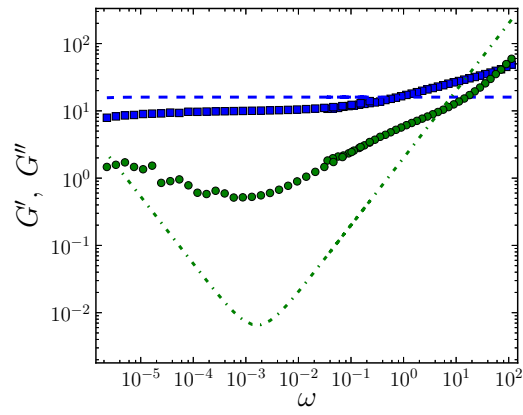

(e) $\phi_{\text {eff }}=0.641$

Figure 3: Linear rheology measurements for different effective volume fractions. (Blue squares) experimental storage moduli $G^{\prime}(\omega)$ from Ref. [25]; (dashed blue line) fit with the WM-type model (see text). (Green dots) experimental loss moduli $G^{\prime \prime}(\omega)$; (dashed green line) fit with the WM-type model. The fitting parameters, as well as the stress and frequency units, are identical to those used in Fig. 2. 
where $p$ is the pressure, and with the postulate of incompressibility,

$$
0=\nabla \cdot \boldsymbol{v}
$$

In the considered geometry, the base flow is purely azimuthal and has no dependence on $\theta$ or $z$, in cylindrical coordinates. It follows from Eq. 44 that the total shear stress must satisfy

$$
\Sigma(r)=\Sigma\left(r_{i}\right) \frac{r_{i}^{2}}{r^{2}}
$$

Inversion of Eqs. 42 and 43 yields, for $\dot{\gamma}\left(r_{i}\right)>0$,

$\dot{\gamma}^{\star}(r)=\frac{1}{2 \alpha \eta_{s}}\left[\alpha \tau_{\alpha} \Sigma(r)-\eta_{0}-\eta_{s}+\sqrt{\left(\eta_{0}+\eta_{s}-\alpha \tau_{\alpha} \Sigma(r)\right)^{2}+4 \eta_{s} \alpha \tau_{\alpha} \Sigma(r)}\right]$,

a profile of which is plotted in Fig. 4 for a particular applied shear rate. Since $\dot{\gamma}^{\star}(r) \equiv v_{\theta}^{\star \prime}-\frac{v_{\theta}^{\star}}{r}$, we can solve numerically for the velocity profile $v_{\theta}(r)$. It is perhaps worth indicating that an analytical solution exists for $\eta_{s}=0$ :

$$
v_{\theta}^{\star}(r)=\frac{r}{\alpha \tau_{\alpha}} \ln \sqrt{\frac{v_{\sigma} \alpha^{-1}-\Sigma(r)}{v_{\sigma} \alpha^{-1}-\Sigma(r)}} .
$$

Incidentally, note that the quality of the approximation $\eta_{s}=0$ is not fixed by the inequality $\eta_{s} \ll \eta_{0}$, but by the more stringent condition $\eta_{s} \ll \frac{\eta_{0}}{1+\alpha \tau_{\alpha}\left|\dot{\gamma}^{\star}\right|}$.

\section{Shear-Thinning suppresses the visco-elastic instability}

\subsection{The visco-elastic instability}

For almost one century, it has been known that the inertial flow of Newtonian liquids is prone to a centrifugal instability at large Reynolds numbers (or, more precisely, at large Taylor numbers), whereby counter-rotating vortices, known as "Taylor vortices", appear and break the full cylindrical symmetry of the base flow [26, 27]. Shear-thinning fluids are also prone to this instability [28], which may even be enhanced, owing to shear-thinning, for polymeric solutions [29].

This type of instability is driven by inertia and opposed by viscosity. In dense colloidal suspensions, the vanishing Reynolds number precludes it. Yet, there exists a distinct type of instability, which does not require inertia: the so called visco-elastic instability was first analysed by Muller, Larson and Shaqfeh [3, 30, 31] and has notably been observed in polymer solutions [3] 


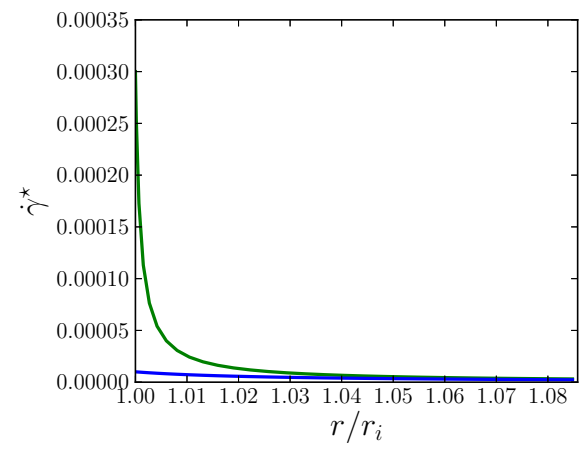

Figure 4: Shear rate profile of the base flow in the Taylor-Couette cell, with model parameters corresponding to $\phi_{\text {eff }}=0.626$, for imposed (nondimensional) shear rates at the rotor (blue) $\dot{\gamma}\left(r_{i}\right)=10^{-5}$ and (green) $\dot{\gamma}\left(r_{i}\right)=$ $3 \cdot 10^{-4}$.

and (semi-dilute) worm-like micellar solutions [4], in Taylor-Couette, coneand-plate and parallel-plate rheometers (for a review, see Ref. [32]). To grasp the importance of this finding, one should remember that, for decades, scientists have measured the rheological properties of polymer solutions and polymer melts in such setups, under the assumption of a well-defined (base) flow.

The precise mechanism driving the instability is still, to some extent, unsettled, but it is clear that curved streamlines and material elasticity (i.e., a finite structural relaxation time) are vital for the (linear) instability to develop. Indeed, because of the curvature in, say, a cylindrical setup, the normal stress $\Sigma_{\theta \theta}$ is coupled to the radial velocity component; this can lead to a positive feedback mechanism, whereby a spontaneous radial velocity perturbation alters $\Sigma_{\theta \theta}$, which further amplifies the perturbation [32]. To assess the effect of the material's properties on the instability, Larson used a model featuring a distribution of relaxation times and an adjustable shearthinning exponent, and showed that shear-thinning tends to stabilise the flow, by shifting the unstable region (in parameter space) to larger applied shear rates [33]. Nevertheless, the author noted that "an elastic instability can occur even in highly shear-thinning entangled polymer solutions", in the light of his calculations and experiments. Clearly, we are interested in knowing whether this also holds true for highly concentrated colloidal suspensions: Does the dramatic shear-thinning behaviour of these materials allow for a visco-elastic instability within the experimentally probed range 
of shear rates, according to the model introduced in the previous section?

\subsection{Pseudo-spectral method for linear stability analysis}

To address this question, we analyse the stability of the base flow in Taylor-Couette geometry (see Section 4.6) with respect to linear perturbations. Although many studies have focused only on axisymmetric instabilities, non-axisymmetric modes were shown to be even more unstable in a number of cases [34, 35], so we investigate three-dimensional perturbations, i.e., perturbations $\delta \phi$ with a spatial dependence not only on $r$ and $z$, but also on $\theta$, viz.,

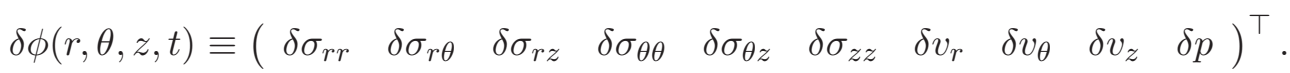

We resort to a pseudo-spectral method.

(i) We start by linearising the equations of the problem, comprising the six constitutive equations (Eqs. 41), the three momentum conservation equations (Eqs. 44), and the incompressibility postulate (Eq. 45), around the base flow so as to obtain a system of linear equations of the form

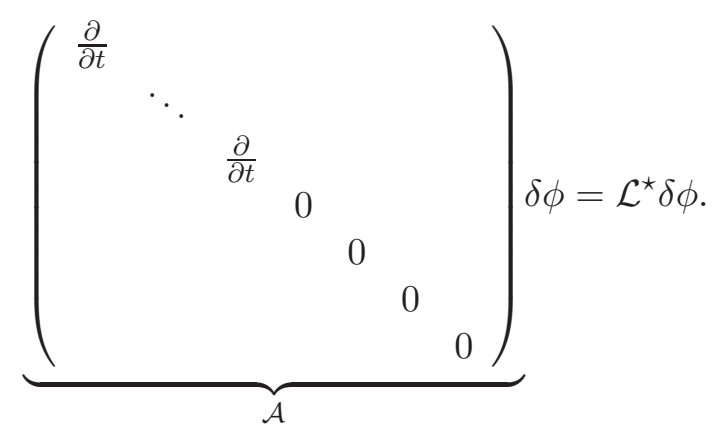

Then we transform the endomorphisms $\mathcal{A}$ and $\mathcal{L}^{\star}$ into matrices, viz., $\mathcal{A} \rightarrow \mathbf{A}$ and $\mathcal{L}^{\star} \rightarrow \mathbf{L}^{\star}$, with the following generic procedure:

(ii) $\delta \phi$ is Fourier-transformed both in the azimuthal direction (wavenumber $m$ ) and in the axial direction (wavenumber $k$ ), viz.,

$$
\delta \phi(r, \theta, z, t)=\sum_{m=-\infty}^{\infty} \sum_{k \in 2 \pi / L_{z} \mathbb{Z}} \delta \phi(r, m, k, t) e^{i m \theta} e^{i k z},
$$

(iii) $\delta \phi$ is discretised along the radial coordinate $r$ by sampling its values at the Chebyshev-Gauss-Lobatto points $r_{n} \equiv 1.0+\frac{\epsilon}{2}\left[1+\cos \left(\pi \frac{n}{M}\right)\right], 0 \leqslant$ $n<M$, for a given $M \in \mathbb{N}^{\star}$. We will typically use $M \approx 2^{6}$ interpolation points across the gap. Radial derivatives $\partial_{r} \delta \phi$ are then written as matrixvector products of the form $D_{r} \cdot \delta \phi[36]$. 
(iv) $\delta \phi$ is Laplace-transformed with respect to time, with Laplace coordinate $s, v i z ., \delta \phi(r, m, k, s)=\int e^{s t} \delta \phi(r, m, k, t) d t$.

Eventually, one obtains the following generalised eigenvalue problem,

$$
\mathbf{A} \delta \phi(r, m, k, s)=s \mathbf{L}^{\star} \delta \phi(r, m, k, s),
$$

where $\mathbf{A}$ and $\mathbf{L}^{\star}$ are $10 M \times 10 M$ matrices, a few lines of which are subsequently substituted for the implementation of the (no-slip) boundary conditions on the velocity.

Let $s^{\star}$ be the maximal growth rate, i.e., the real part of the maximal eigenvalue 3 of Eq. 47 over all possible wavenumbers $m$ and $k$. Then the base flow is (linearly) stable if, and only if, $s^{\star}<0$.

\subsection{Linearised equations}

The linearised constitutive equations read:

$$
\begin{aligned}
\frac{\partial \delta \sigma_{r r}}{\partial t}= & \left(\frac{-1}{\tau^{\star}}-\frac{i m}{r} v_{\theta}^{\star}\right) \delta \sigma_{r r}+\left(2 \sigma_{r \theta}^{\star} \frac{i m}{r}+2 v_{\sigma} \partial_{r}\right) \delta v_{r} \\
\frac{\partial \delta \sigma_{r \theta}}{\partial t}= & \dot{\gamma}^{\star} \delta \sigma_{r r}+\left(\frac{-1}{\tau^{\star}}-\frac{i m}{r} v_{\theta}^{\star}\right) \delta \sigma_{r \theta}+\left[\sigma_{r \theta}^{\star} \frac{i m}{r}+v_{\sigma}\left(\partial_{r}-\frac{1}{r}\right)-\alpha \sigma_{r \theta}^{\star}\left(\partial_{r}-\frac{1}{r}\right)\right] \delta v_{\theta} \\
& +\left[\sigma_{r \theta}^{\star}\left(\partial_{r}+\frac{1}{r}\right)-\partial_{r} \sigma_{r \theta}^{\star}+\sigma_{\theta \theta}^{\star} \frac{i m}{r}+v_{\sigma} \frac{i m}{r}-\alpha \sigma_{r \theta}^{\star} \frac{i m}{r}\right] \delta v_{r} \\
\frac{\partial \delta \sigma_{r z}}{\partial t}= & \left(\frac{-1}{\tau^{\star}}-\frac{i m}{r} v_{\theta}^{\star}\right) \delta \sigma_{r z}+i k v_{\sigma} \delta v_{r}+\left(\sigma_{r \theta}^{\star} \frac{i m}{r}+v_{\sigma} \partial_{r}\right) \delta v_{z} \\
\frac{\partial \delta \sigma_{\theta \theta}}{\partial t}= & 2 \dot{\gamma}^{\star} \delta \sigma_{r \theta}+\left(\frac{-1}{\tau^{\star}}-\frac{i m}{r} v_{\theta}^{\star}\right) \delta \sigma_{\theta \theta}+\left(\frac{2 \sigma_{\theta \theta}^{\star}}{r}-\partial_{r} \sigma_{\theta \theta}^{\star}+\frac{2 v_{\sigma}}{r}-\alpha \sigma_{\theta \theta}^{\star} \frac{i m}{r}\right) \delta v_{r} \\
& +\left[2 \sigma_{r \theta}^{\star}\left(\partial_{r}-1 / r\right)+2 \sigma_{\theta \theta}^{\star} \frac{i m}{r}+2 v_{\sigma} \frac{i m}{r}-\alpha \sigma_{\theta \theta}^{\star}\left(\partial_{r}-\frac{1}{r}\right)\right] \delta v_{\theta} \\
\frac{\partial \delta \sigma_{\theta z}}{\partial t}= & \dot{\gamma}^{\star} \delta \sigma_{r z}+\left(\frac{-1}{\tau^{\star}}-\frac{i m}{r} v_{\theta}^{\star}\right) \delta \sigma_{\theta z}+i k v_{\sigma} \delta v_{\theta}+\left(v_{\sigma} \frac{i m}{r}+\sigma_{\theta \theta}^{\star} \frac{i m}{r}+\sigma_{r \theta}^{\star} \partial_{r}\right) \delta v_{z} \\
\frac{\partial \delta \sigma_{z z}}{\partial t}= & \left(\frac{-1}{\tau^{\star}}-\frac{i m}{r} v_{\theta}^{\star}\right) \delta \sigma_{z z}+2 i k v_{\sigma} \delta v_{z},
\end{aligned}
$$

\footnotetext{
${ }^{3}$ Due to the discretisation, some spurious eigenvalues may pop up in the generalised eigenvalue problem (Eq. 47), but they can easily be eliminated because, unlike their physical counterparts, they vary with the number of discretisation points.
} 
where $\tau^{\star} \equiv \frac{\tau_{\alpha}}{1+\alpha \tau_{\alpha} \dot{\gamma}^{\star}}$, while the linearised momentum conservation equations are

$$
\begin{aligned}
0= & \left(\partial_{r}+\frac{1}{r}\right) \delta \sigma_{r r}+\frac{i m}{r} \delta \sigma_{r \theta}+i k \delta \sigma_{r z}-\frac{1}{r} \delta \sigma_{\theta \theta}-\partial_{r} \delta p \\
& +\eta_{s}\left[\partial_{r}^{2}+\frac{1}{r} \partial_{r}-\frac{1}{r^{2}}-\frac{m^{2}}{r^{2}}-k^{2}\right] \delta v_{r}-2 \eta_{s} \frac{i m}{r^{2}} \delta v_{\theta} \\
0= & \left(\partial_{r}+\frac{2}{r}\right) \delta \sigma_{r \theta}+\frac{i m}{r} \delta \sigma_{\theta \theta}+i k \delta \sigma_{\theta z}-\frac{i m}{r} \delta p \\
& +\eta_{s}\left[\partial_{r}^{2}+\frac{1}{r} \partial_{r}-\frac{m^{2}}{r^{2}}-\frac{1}{r^{2}}-k^{2}\right] \delta v_{\theta}+2 \eta_{s} \frac{i m}{r^{2}} \delta v_{r} \\
0 & \left(\partial_{r}+\frac{1}{r}\right) \delta \sigma_{r z}+\frac{i m}{r} \delta \sigma_{\theta z}+i k \delta \sigma_{z z}-i k \delta p, \\
& +\eta_{s}\left[\partial_{r}^{2}+\frac{1}{r} \partial_{r}-\frac{m^{2}}{r^{2}}-k^{2}\right] \delta v_{z}
\end{aligned}
$$

and incompressibility states that

$$
0=\left(\partial_{r}+\frac{1}{r}\right) \delta v_{r}+\frac{i m}{r} \delta v_{\theta}+i k \delta v_{z}
$$

When the shear-thinning parameter $\alpha$ vanishes, these linear equations reduce to those derived by Avgousti and Beris [34] for an Oldroyd-B fluid; as a matter of fact, we have spotted slight differences with Ref. [34], which we believe are typos in that publication.

These equations involve three non-dimensional quantities: the shearthinning parameter $\alpha$, the "bare Weissenberg" number $\tau_{\alpha} \dot{\gamma}^{\star}$, and the relative Newtonian viscosity $\eta_{s} / \eta_{0}$, on top of the (fixed) relative curvature $\epsilon$.

\subsection{Linear stability analysis: general trends and application to dense sus- pensions}

5.4.1. Consistency of the algorithm for $\alpha \rightarrow 0, \eta_{s} \rightarrow 0$ and influence of the shear rate

To check the validity of our algorithm, we have first set the shear-thinning parameter $\alpha$ and the Newtonian viscosity $\eta_{s}$ to values close to zero. A conventional Upper Convected Maxwell (UCM) model should then be recovered, in which case, following Ref. [30], some axisymmetric modes (at $m=0$ ) become unstable at sufficiently large applied shear rates. This is indeed the case in our simulations, as soon as the shear rate grows larger than a critical shear rate comparable to that reported in Ref. [30]. However, we also 


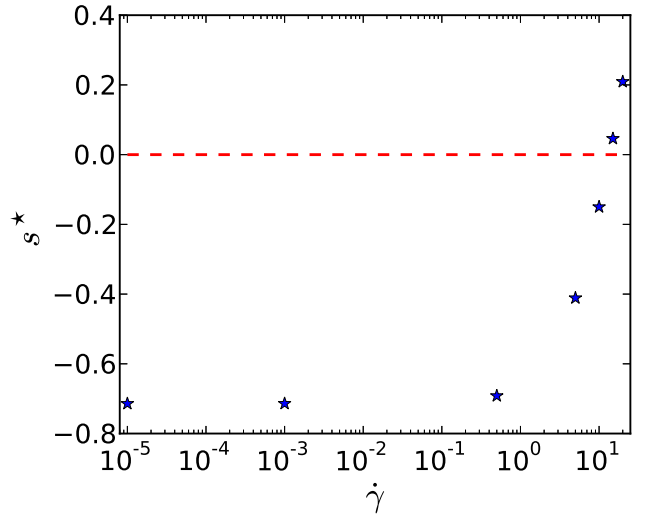

(a) $s^{\star}$ vs. applied shear rate $\dot{\gamma}$ for vanishing Newtonian viscosity $\left(\eta_{s} / \eta_{0}=10^{-6}\right)$ (UCM model).

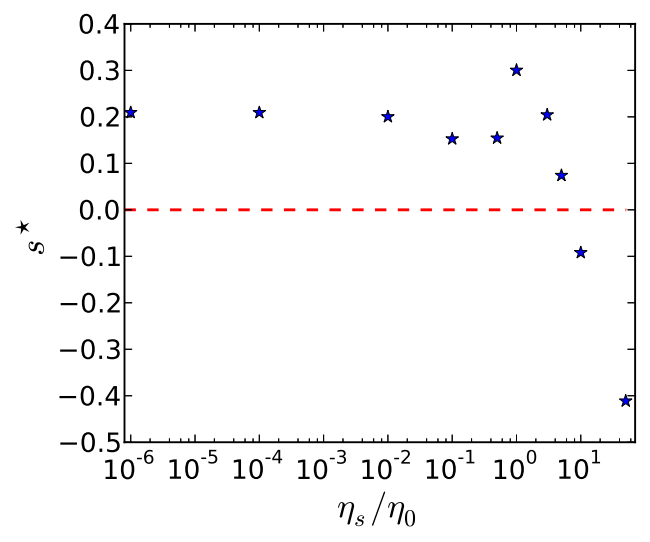

(b) $s^{\star}$ vs. $\eta_{s}$, at fixed shear rate $(\dot{\gamma}=20)$.

Figure 5: Maximal growth rate $s^{\star}$ (over all modes) in the limit of a nonshear-thinning fluid, $\alpha=3 \cdot 10^{-4}, \tau_{\alpha}=1.4$. At $\dot{\gamma}=20$, the most unstable modes are at $m \simeq 1, k \simeq 70$. The dashed red line separates stable base flows (below the line) from unstable ones (above).

observe that some non-axisymmetric modes, associated to small azimuthal wavenumbers $m=\mathcal{O}(1)$, are even more unstable than the axisymmetric ones, in accordance with the literature [34]. Figure 5 shows the increase of the growth rate $s^{\star}$ of the most unstable mode with the shear rate $\dot{\gamma}$ measured at the inner cylinder (or the "bare Weissenberg" number $\tau_{\alpha} \dot{\gamma}$ ).

\subsubsection{Stabilising effect of the Newtonian viscosity}

Including a Newtonian contribution to the stress, via a finite value of the viscosity $\eta_{s}$, tends to stabilise the flow, as shown in Fig. 5b. This stabilising role has already been reported in the literature on the Oldroyd-B model (i.e., for $\alpha=0$ ) [34, 32]; we find that it holds true for shear-thinning fluids, i.e., when $\alpha$ departs from zero (see Fig. 6) ).

\subsubsection{Stabilisation through shear-thinning}

For the values of $\alpha$ of interest here, shear-thinning strongly suppresses the instability. Figures 78 show that, for the model parameters corresponding to the colloidal suspension at $\phi_{\text {eff }}=0.519$, but with vanishing Newtonian viscosity, the base flow lies deep within the stable region, whereas, in the 


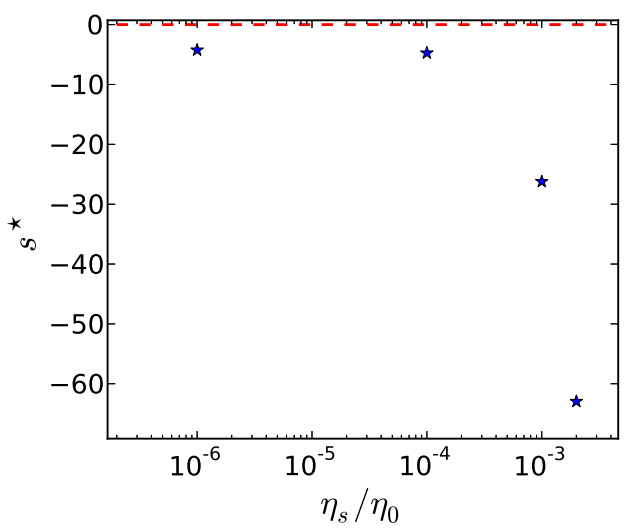

Figure 6: Dependence of the maximal growth rate $s^{\star}$ on the Newtonian viscosity $\eta_{s}$, for a shear-thinning fluid with the following parameters: $\alpha=$ $7.7, \tau_{\alpha}=1.4, \dot{\gamma}=20$. (The growth rates vary only weakly with $k$ and $m$ for these parameters; only modes $m \leqslant 250$ are considered here).

absence of shear-thinning, i.e., were $\alpha$ vanishingly small, a linear instability would develop, with a peak of instability around $k \simeq 70, m \simeq 1$.

We should however mention the existence of a limited range of values of $\alpha$, outside the experimentally relevant window for dense colloidal suspensions, namely, $0.3 \lesssim \alpha \lesssim 3$ and marked by red vertical bars on Fig. 7, which display unstable modes associated to abnormally large growth rates. These modes are located in a very different region of the $(k, m)$-plane, namely $k \approx 0$ and $m \gg 1$. Although these perturbations have reasonable shapes in space, it is plausible that they are actually unphysical, but we do not know whether they are intrinsic in our WM-model or whether they arise because of artifacts in the (well established) numerical method. In the following, we concentrate on the experimentally relevant range of parameters.

\subsubsection{Linear stability analysis of dense colloidal suspensions}

We now specifically consider the model parameters used to fit the rheology of dense colloidal suspensions, (see Table 1).

Consistently with the strongly stabilising effect of shear-thinning described in the previous section (Section 5.4.3), we have numerically ascertained the stability of the base flows corresponding to the suspension at $\phi=0.519$ for rescaled shear rates $\dot{\gamma}=10^{-4}, 10^{-2}, 10^{-1}$, at $\phi_{\text {eff }}=0.626$ for $\dot{\gamma}=5 \cdot 10^{-6}, 5 \cdot 10^{-4}, 5 \cdot 10^{-3}, 10^{-2}$ in the experimental range, as well as that with effective volume fraction $\phi_{\text {eff }}=0.641$ at $\dot{\gamma}=10^{-8}, 10^{-6}, 10^{-4}$. 


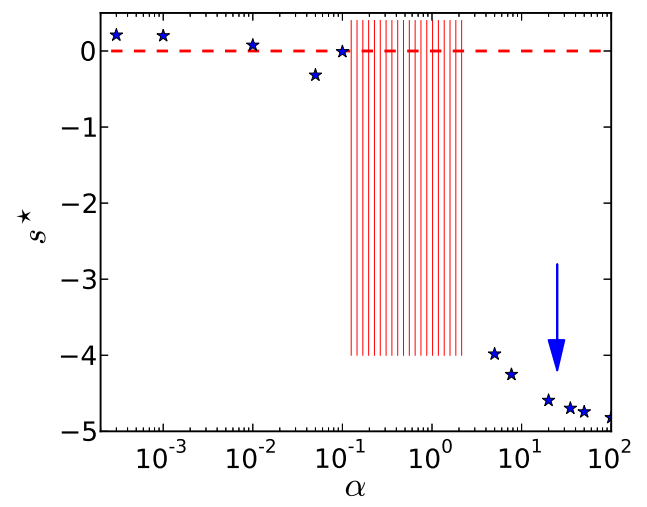

Figure 7: Dependence of the maximal growth rate $s^{\star}$ on the shear-thinning parameter $\alpha$, for $\eta_{s} / \eta_{0}=10^{-6}, \tau_{\alpha}=1.4, \dot{\gamma}=20$. The arrow points to the typical values of $\alpha$ of interest here. See the text for the description of the red vertical bars.

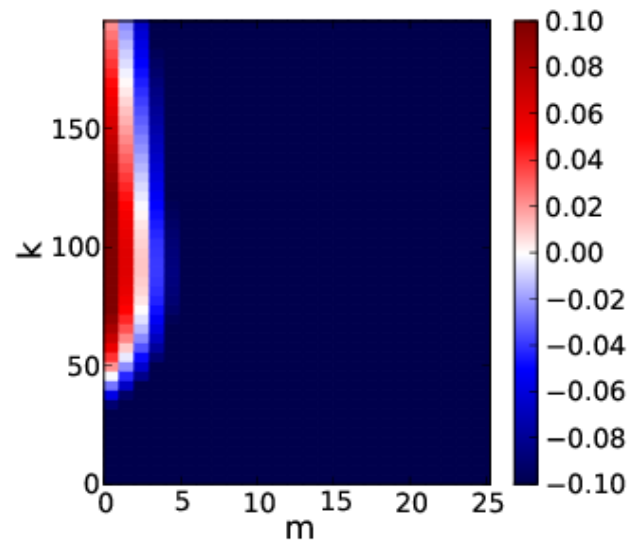

(a) $\alpha=3 \cdot 10^{-4}$

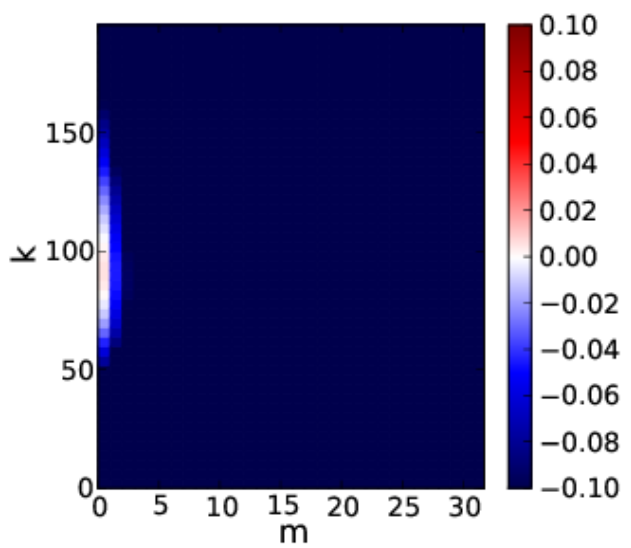

(b) $\alpha=10^{-2}$

Figure 8: Colour maps of the maximal growth rates associated with each pair of wavenumbers $(m, k)$ for two distinct, but small, values of $\alpha$ (see captions), at $\dot{\gamma}=20$, with negligible Newtonian viscosity $\left(\eta_{s} / \eta_{0}=10^{-6}\right)$. Stable modes appear in dark blue. 
The relative contribution of the Newtonian viscosity to the stabilisation of the flow echoes the contribution of the Newtonian stress to the total stress: at high densities and low shear rates, the effect of the Newtonian viscosity is negligible. Admittedly, the more acute shear-thinning displayed by the WM models fitted to the densest suspensions results in an enhanced stability compared to the experimental systems; however the fact that they lie so deep in the stable region suggests that the materials they model, albeit somewhat less shear-thinning, are also stable with respect to the considered perturbations. This is further supported by the observed stability of the system at $\phi=0.519$, which is both less shear-thinning than the others and very well described by the WM model.

\subsection{Rationalisation with the Pakdel-McKinley criterion}

Before we conclude, it is interesting to note that the stabilising effects of shear-thinning and of the Newtonian contribution are in line with the viscoelastic stability criterion proposed by Pakdel and McKinley in 1996 [37, 38] (less general versions of the criterion can be found in earlier publications [30]). On the basis of a dimensional analysis of generic visco-elastic constitutive equations, these authors introduced a dimensionless number, written $\mathcal{P}$ here, for inertialess visco-elastic instabilities in curved geometries in analogy to the classical Taylor number for inertial instabilities, and propounded the idea that the base flow is susceptible to an instability if this dimensional number exceeds a given threshold of order unity. The Pakdel-McKinley number reads

$$
\mathcal{P} \equiv \frac{l_{p}}{\mathcal{R}} \frac{N_{1}}{\Sigma}
$$

where $l_{p} \equiv v_{\theta} \tau$ is the typical distance travelled by a material substructure (e.g., a polymer chain) along the base-flow streamline while relaxing, $\mathcal{R}$ is the radius of curvature of the streamline, $N_{1}$ is the first normal-stress-difference and $\Sigma$ is the shear stress. For a UCM model, the ratio $\frac{N_{1}}{\Sigma}$ is simply the Weissenberg number and measures the "anisotropy of the normal forces", as phrased by Morozov and van Saarloos [38]. But even with the present WM model, which is somewhat more complicated, the criterion based on $\mathcal{P}$ seems to capture the observed trends: a Newtonian contribution to the stress increases $\Sigma$ without altering $N_{1}$, thereby reducing $\mathcal{P}$ and stabilising the flow. On the other hand, an enhanced propensity to shear-thinning results in a decrease of $N_{1}$ as $\left(1+\alpha \tau_{\alpha} \dot{\gamma}\right)^{-2}$, while $\Sigma$ only decreases as $\left(1+\alpha \tau_{\alpha} \dot{\gamma}\right)^{-1}$ (for $\eta_{s} \rightarrow 0$ ); as a result, $\mathcal{P}$ is reduced, which also explains the enhanced stability of the flow. 
The Newtonian stress contribution and the propensity to shear-thinning thus act in synergy, but, for the rheology of the very dense colloidal suspensions of interest here, our results indicate that the impact of shear-thinning quantitatively prevails over that of the former.

\section{Discussion and Outlook}

In conclusion, we have made use of, and partially extended, a general theoretical framework based on projections onto density modes, to describe the rheology of dense colloidal suspensions in the vicinity of the glass transition. Contrary to the previous theoretical works, which focused on strictly homogeneous flows, special attention was paid to the fate of spatial inhomogeneities. However, the intricacy of the formalism forced us to resort to particularly strong approximations before we could study the stability of the flow in curved (Taylor-Couette) geometry. At the expense of these approximations, constitutive equations falling in the White-Metzner class were obtained; the resulting model was shown to capture the shear-thinning properties of the material and to be reasonably consistent with experimental measurements of the linear rheology and steady-state rheology of the suspensions, although not in a strictly quantitative way. Eventually, we analysed the stability of the visco-elastic flow and brought evidence that, in the experimental range of shear rates, shear-thinning (and to a much lesser extent the Newtonian stress contribution) strongly stabilise the flow. This may explain why visco-elastic instabilities have been observed in a variety of visco-elastic fluids, but not in the dense suspensions under consideration here: the flow strains and destroys the microstructure of the material, so much so that, if a material volume is displaced by a perturbation, the memory of the stress that it carries, through its microstructure, is suppressed by the flow too quickly to allow the possibility of a feedback mechanism.

Acknowledgments. We thank Miriam Siebenbürger for providing us with the experimental rheological data. AN thanks Alexander Morozov for introducing him to the pseudo-spectral method, Fabian Frahsa for enlightening

discussions, and the DFG-funded research unit FOR 1394 project P3 for partial funding of his stay at the University of Konstanz. 


\section{References}

[1] P. Pusey and W. Van Megen, "Observation of a glass transition in suspensions of spherical colloidal particles," Physical review letters, vol. 59, no. 18, p. 2083, 1987.

[2] C. Amann, M. Siebenbürger, M. Krüger, F. Weysser, M. Ballauff, and M. Fuchs, "Overshoots in stress-strain curves: Colloid experiments and schematic mode coupling theory," Journal of Rheology, vol. 57, no. 1, p. 149, 2013.

[3] S. Muller, R. Larson, and E. Shaqfeh, "A purely elastic transition in taylor-couette flow," Rheologica Acta, vol. 28, no. 6, pp. 499-503, 1989.

[4] M. Fardin, T. Ober, V. Grenard, T. Divoux, S. Manneville, G. McKinley, and S. Lerouge, "Interplay between elastic instabilities and shearbanding: three categories of Taylor-Couette flows and beyond," Soft Matter, 2012.

[5] A. Einstein, "Eine neue bestimmung der moleküldimensionen," Annalen der Physik, vol. 324, no. 2, pp. 289-306, 1906.

[6] G. Batchelor, "The effect of brownian motion on the bulk stress in a suspension of spherical particles," Journal of Fluid Mechanics, vol. 83, no. 01, pp. 97-117, 1977.

[7] J. F. Brady, "Model hard-sphere dispersions: statistical mechanical theory, simulations, and experiments," Current Opinion in Colloid \& Interface Science, vol. 1, no. 4, pp. 472-480, 1996.

[8] J. M. Brader, "Nonlinear rheology of colloidal dispersions," Journal of Physics: Condensed Matter, vol. 22, no. 36, p. 363101, 2010.

[9] U. Bengtzelius, W. Götze, and A. Sjolander, "Dynamics of supercooled liquids and the glass transition," Journal of Physics C: Solid State Physics, vol. 17, no. 33, pp. 5915-5934, 1984.

[10] L. Sjögren, "Kinetic theory of current fluctuations in simple classical liquids," Physical Review A, vol. 22, pp. 2866-2882, 1980.

[11] M. Fuchs and M. Cates, "Theory of Nonlinear Rheology and Yielding of Dense Colloidal Suspensions," Physical Review Letters, vol. 89, no. 24, 2002. 
[12] J. Brader, M. Cates, and M. Fuchs, "First-principles constitutive equation for suspension rheology," Physical Review E, vol. 86, no. 2, p. 021403, 2012.

[13] K. Miyazaki, D. Reichman, and R. Yamamoto, "Supercooled liquids under shear: Theory and simulation," Physical Review E, vol. 70, no. 1, p. 011501, 2004.

[14] C. Amann, M. Siebenbürger, M. Ballauff, and M. Fuchs, "Nonlinear rheology of glass-forming colloidal dispersions: transient stress-strain relations from anisotropic mode coupling theory and thermosensitive microgels," Journal of Physics: Condensed Matter, vol. 27, no. 19, p. 194121, 2015.

[15] J. Brader, T. Voigtmann, M. Fuchs, R. Larson, and M. Cates, "Glass rheology: From mode-coupling theory to a dynamical yield criterion.," Proceedings of the National Academy of Sciences of the United States of America, vol. 106, no. 36, pp. 15186-91, 2009.

[16] J. White and A. Metzner, "Development of constitutive equations for polymeric melts and solutions," Journal of Applied Polymer Science, vol. 7, no. 5, pp. 1867-1889, 1963.

[17] M. Siebenbürger, M. Fuchs, and M. Ballauff, "Core-shell microgels as model colloids for rheological studies," Soft Matter, vol. 8, no. 15, pp. 4014-4024, 2012.

[18] T. Voigtmann, "Nonlinear glassy rheology," Current Opinion in Colloid 8 Interface Science, vol. 19, no. 6, pp. 549-560, 2014.

[19] H. Risken, The Fokker-Planck Equation. Methods of Solution and Applications. 1989.

[20] M. Fuchs and M. Cates, "A mode coupling theory for Brownian particles in homogeneous steady shear flow," Journal of Rheology, vol. 53, no. 4, p. $957,2009$.

[21] J. Wu and J. Cao, "Gaussian factorization of hydrodynamic correlation functions and mode-coupling memory kernels," Physical Review E, vol. 67, no. 6, p. 061116, 2003.

[22] R. Besseling, L. Isa, P. Ballesta, G. Petekidis, M. Cates, and W. Poon, "Shear banding and flow-concentration coupling in colloidal glasses," Physical Review Letters, vol. 105, no. 26, p. 268301, 2010. 
[23] H. Jin, K. Kang, K. H. Ahn, and J. K. Dhont, "Flow instability due to coupling of shear-gradients with concentration: non-uniform flow of (hard-sphere) glasses," Soft Matter, vol. 10, no. 47, pp. 9470-9485, 2014.

[24] S. Papenkort and T. Voigtmann, "Channel flow of a tensorial shearthinning maxwell model: Lattice boltzmann simulations," The Journal of Chemical physics, vol. 140, no. 16, p. 164507, 2014.

[25] M. Siebenbürger, M. Fuchs, H. Winter, and M. Ballauff, "Viscoelasticity and shear flow of concentrated, noncrystallizing colloidal suspensions: Comparison with mode-coupling theory," Journal of Rheology (1978present), vol. 53, no. 3, pp. 707-726, 2009.

[26] G. Taylor, "Stability of a viscous liquid contained between two rotating cylinders," Proceedings of the Royal Society of London. Series A, vol. 102, no. 718, pp. 541-542, 1923.

[27] M. A. Fardin, C. Perge, and N. Taberlet, "the hydrogen atom of fluid dynamics" - introduction to the taylor-couette flow for soft matter scientists," Soft Matter, vol. 10, pp. 3523-3535, 2014.

[28] O. Coronado-Matutti, P. S. Mendes, and M. Carvalho, "Instability of inelastic shear-thinning liquids in a couette flow between concentric cylinders," Journal of fluids engineering, vol. 126, no. 3, pp. 385-390, 2004.

[29] R. Larson, "Taylor-couette stability analysis for a doi-edwards fluid," Rheologica Acta, vol. 28, no. 6, pp. 504-510, 1989.

[30] R. Larson, E. Shaqfeh, and S. Muller, "A purely elastic instability in taylor-couette flow," Journal of Fluid Mechanics, vol. 218, pp. 573-600, 1990.

[31] E. Shaqfeh, S. Muller, and R. Larson, "The effects of gap width and dilute solution properties on the viscoelastic Taylor-Couette instability," Journal of Fluid Mechanics, vol. 235, no. -1, pp. 285-317, 1992.

[32] E. Shaqfeh, "Purely elastic instabilities in viscometric flows," Annual Review of Fluid Mechanics, vol. 28, no. 1, pp. 129-185, 1996.

[33] R. Larson, "The effect of fluid rheology on the elastic Taylor-Couette instability," Journal of Non-Newtonian Fluid Mechanics, vol. 51, no. 2, pp. 195-225, 1994. 
[34] M. Avgousti, "Non-axisymmetric modes in viscoelastic taylor-couette flow," Journal of Non-Newtonian Fluid Mechanics, vol. 50, no. 2-3, pp. 225-251, 1993.

[35] A. Nicolas and A. Morozov, "Nonaxisymmetric Instability of ShearBanded Taylor-Couette Flow," Physical Review Letters, vol. 108, no. 8, 2012.

[36] R. Peyret, Spectral methods for incompressible viscous flow, vol. 148. Springer Science \& Business Media, 2002.

[37] P. Pakdel and G. McKinley, "Elastic instability and curved streamlines," Physical Review Letters, vol. 77, no. 12, p. 2459, 1996.

[38] A. N. Morozov and W. van Saarloos, "An introductory essay on subcritical instabilities and the transition to turbulence in visco-elastic parallel shear flows," Physics Reports, vol. 447, no. 3, pp. 112-143, 2007.

\section{Appendix A. Operator formulation of the switch to the auxiliary frame}

In Section 2.2, the switch to the auxiliary frame at a specific point was presented as a change of coordinates. However, it may also be conducted with the operator formalism. Indeed, consider an auxiliary system (denoted by tildes) with the following Smoluchowski operator

$$
\tilde{\Omega}^{\dagger}(\Gamma, t) \equiv \sum_{i=1}^{N}\left[\partial_{i}+\boldsymbol{F}_{\boldsymbol{i}}(\Gamma)+\tilde{\boldsymbol{v}}\left(\boldsymbol{r}_{\boldsymbol{i}}, t\right)\right] \cdot \partial_{i},
$$

where

$$
\tilde{\boldsymbol{v}}(\boldsymbol{r}, t) \equiv \boldsymbol{v}^{\text {solv }}(\boldsymbol{r}, t)-\boldsymbol{v}^{\text {solv }}\left(\boldsymbol{r}_{o}(t), t\right) .
$$

As in Section 2.2, $\boldsymbol{r}_{o}(t)$ is the position (given by Eq. 8) of the "material point" advected by the solvent flow field, with position $\boldsymbol{r}_{o}$ at $t_{o}$.

In the auxiliary system, an observable $\tilde{g}(\boldsymbol{r}, \Gamma ; t)$ evolves as

$$
\partial_{t} \tilde{g}(\boldsymbol{r}, \Gamma ; t)=\tilde{\Omega}^{\dagger}(\Gamma, t) \tilde{g}(\boldsymbol{r}, \Gamma ; t) .
$$

Evaluating an observable in this auxiliary system is therefore equivalent to evaluating it in the auxiliary (denoted by primes) of Section $2.2 . \tilde{g}(\boldsymbol{r}, \Gamma ; t)$ and $\left.g^{\prime}\left(\boldsymbol{r}, \Gamma^{\prime} ; t\right)\right|_{\Gamma^{\prime}=\Gamma}$ have the same time derivative $\tilde{\Omega}^{\dagger}(\Gamma, t)=\left.\Omega^{\dagger^{\prime}}\left(\Gamma^{\prime}, t\right)\right|_{\Gamma^{\prime}=\Gamma}$, and they coincide at $t_{o}$, so they are equal at all times. 
The formal solution of Eq. A.1 is

$$
\begin{aligned}
\tilde{g}(\boldsymbol{r}, \Gamma ; t) & =e_{-}^{\int_{0}^{t} \tilde{\Omega}^{\dagger}(\Gamma, s) d s} \tilde{g}(\boldsymbol{r}, \Gamma) \\
& =e_{-}^{\int_{0}^{t} \tilde{\Omega}^{\dagger}(\Gamma, s) d s} g(\boldsymbol{r}, \Gamma) .
\end{aligned}
$$

Using the shorthand $A^{\dagger}(t)$ for $\boldsymbol{v}^{\text {solv }}\left(\boldsymbol{r}_{o}(t), t\right) \cdot \sum_{i=1}^{N} \partial_{i}$, we observe that $A^{\dagger}(t)$ commutes with all $\Omega^{\dagger}(s)$. Indeed,

$$
\begin{aligned}
\Omega^{\dagger}(s) A^{\dagger}(t) g & =\sum_{i=1}^{N}\left[\partial_{i}+\boldsymbol{F}_{\boldsymbol{i}}(\Gamma)+\boldsymbol{v}^{\text {solv }}\left(\boldsymbol{r}_{\boldsymbol{i}}, s\right)\right] \cdot \partial_{i}\left[\boldsymbol{v}^{\text {solv }}\left(\boldsymbol{r}_{o}(t), t\right) \cdot \sum_{j=1}^{N} \partial_{j} g\right] \\
& =\boldsymbol{v}^{\text {solv }}\left(\boldsymbol{r}_{o}(t), t\right) \cdot \sum_{i=1}^{N} \sum_{j=1}^{N}\left[\partial_{j} \partial_{i} g+\partial_{j}\left(\boldsymbol{F}_{\boldsymbol{i}} \cdot \partial_{i} g\right)+\partial_{j} \boldsymbol{v}^{\text {solv }}\left(\boldsymbol{r}_{\boldsymbol{i}}, s\right) \partial_{i} g\right], \\
& =A^{\dagger}(t) \Omega^{\dagger}(s) g,
\end{aligned}
$$

where we have made use of $\partial_{j} \boldsymbol{v}^{\text {solv }}\left(\boldsymbol{r}_{\boldsymbol{j}}, s\right)=0$ and $\sum_{i=1}^{N} \boldsymbol{F}_{\boldsymbol{i}}=0$, hence $\sum_{j=1}^{N} \sum_{i=1}^{N} \partial_{j} \boldsymbol{F}_{\boldsymbol{i}}=\mathbf{0}$. It follows that

$$
\begin{aligned}
\tilde{g}(\boldsymbol{r}, \Gamma ; t) & =e_{-}^{-\int_{0}^{t} d s \boldsymbol{v}^{\mathrm{solv}}}\left(\boldsymbol{r}_{o}(s), s\right) \cdot \sum_{i=1}^{N} \partial_{i} e_{-}^{\int_{0}^{t} \Omega^{\dagger}(\Gamma, s) d s} g(\boldsymbol{r}, \Gamma), \\
& =e_{-}^{-\int_{0}^{t} d s \boldsymbol{v}^{\mathrm{solv}}\left(\boldsymbol{r}_{o}(s), s\right) \cdot \sum_{i=1}^{N} \partial_{i}} g(\boldsymbol{r}, \Gamma ; t)
\end{aligned}
$$

and, if $g$ does not depend intrinsically on space,

$$
\tilde{g}(\boldsymbol{r}, \Gamma ; t)=e_{-}^{\int_{0}^{t} d s \boldsymbol{v}^{\mathrm{solv}}\left(\boldsymbol{r}_{o}(s), s\right) \cdot \partial_{\boldsymbol{r}}} g(\boldsymbol{r}, \Gamma ; t) .
$$

In particular, the fluctuation advection term emerges when the equation is differentiated with respect to time.

$$
\partial_{t} g(\boldsymbol{r}, \Gamma ; t)=e_{-}^{-\int_{0}^{t} d s \boldsymbol{v}^{\mathrm{solv}}\left(\boldsymbol{r}_{o}(s), s\right) \cdot \partial_{\boldsymbol{r}}} \partial_{t} \tilde{g}(\boldsymbol{r}, \Gamma ; t)-\boldsymbol{v}^{\mathrm{solv}}\left(\boldsymbol{r}_{o}(t), t\right) \cdot \partial_{\boldsymbol{r}} g(\boldsymbol{r}, \Gamma ; t) .
$$

This equation agrees with Eq. 14 


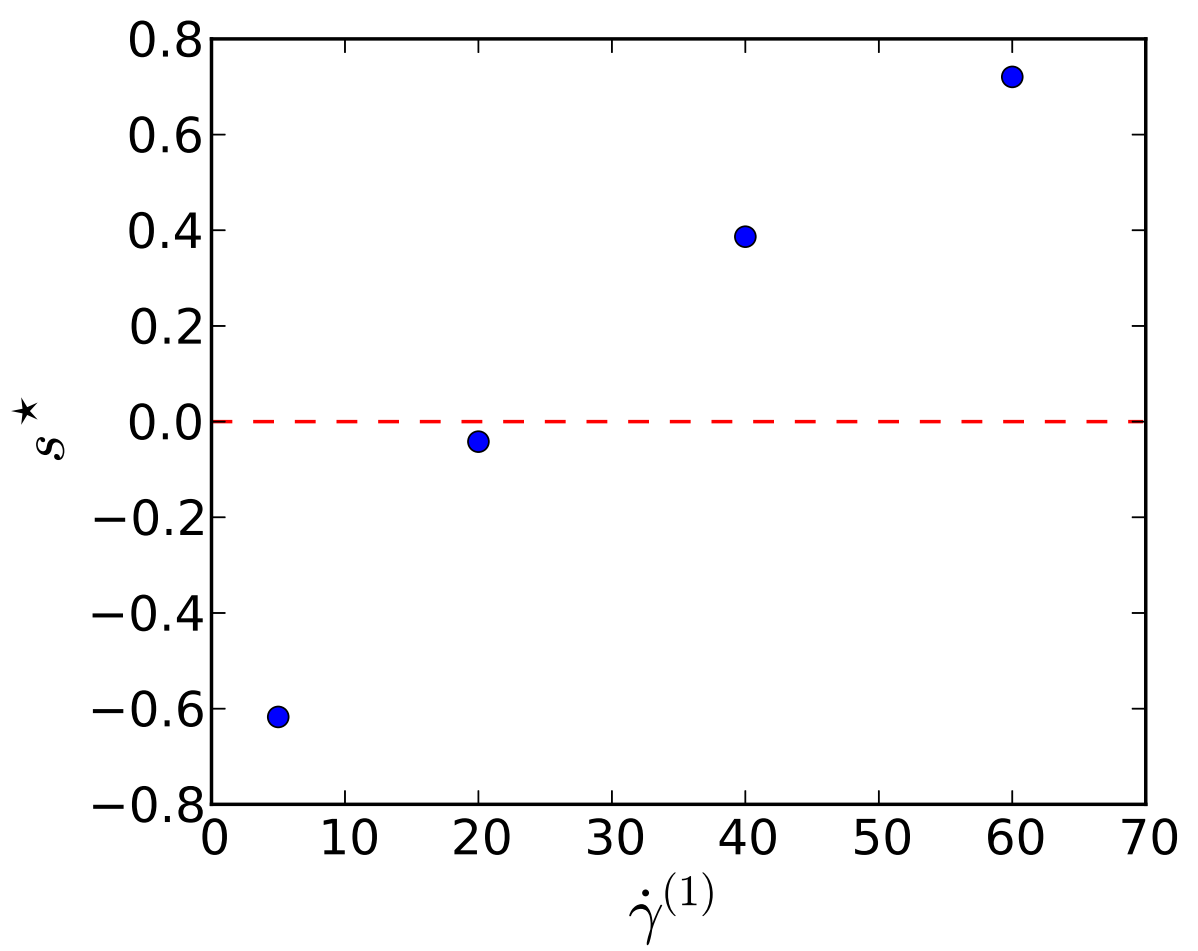




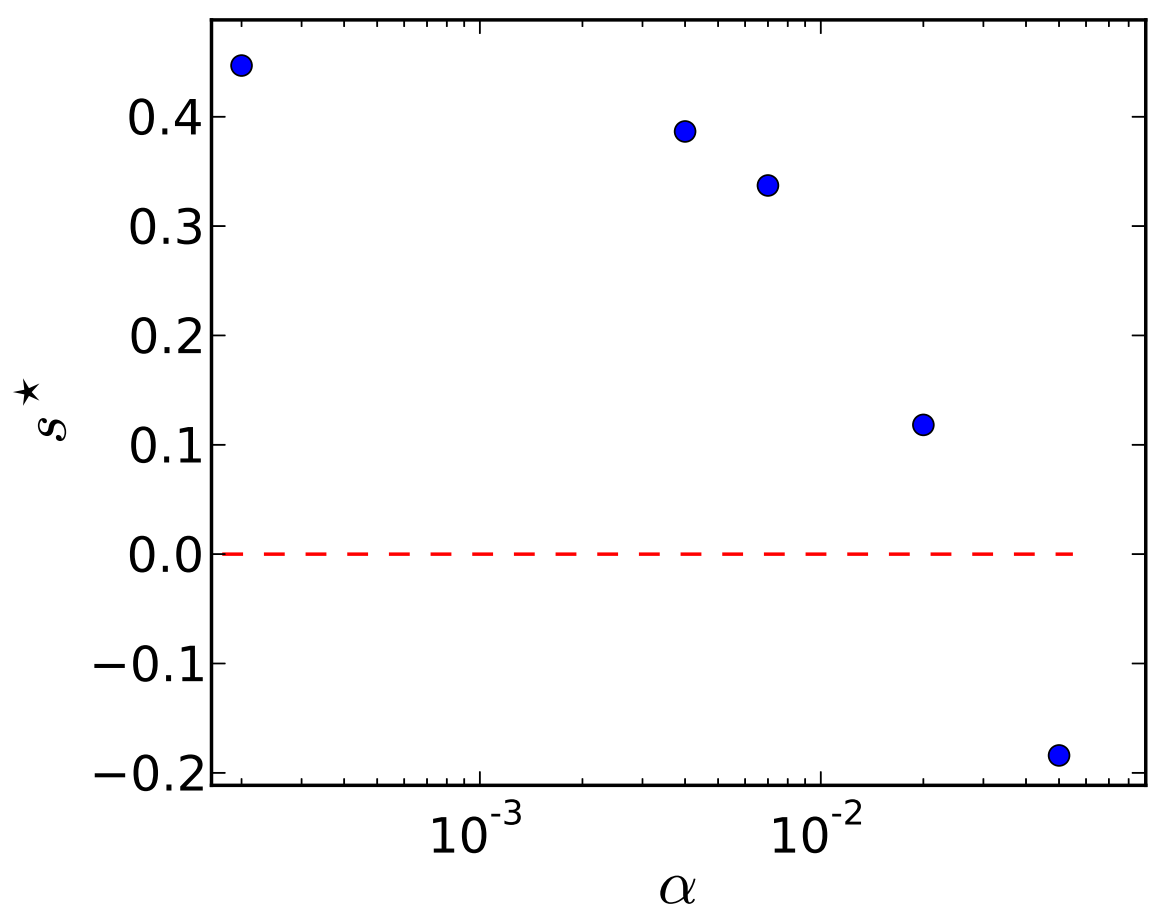




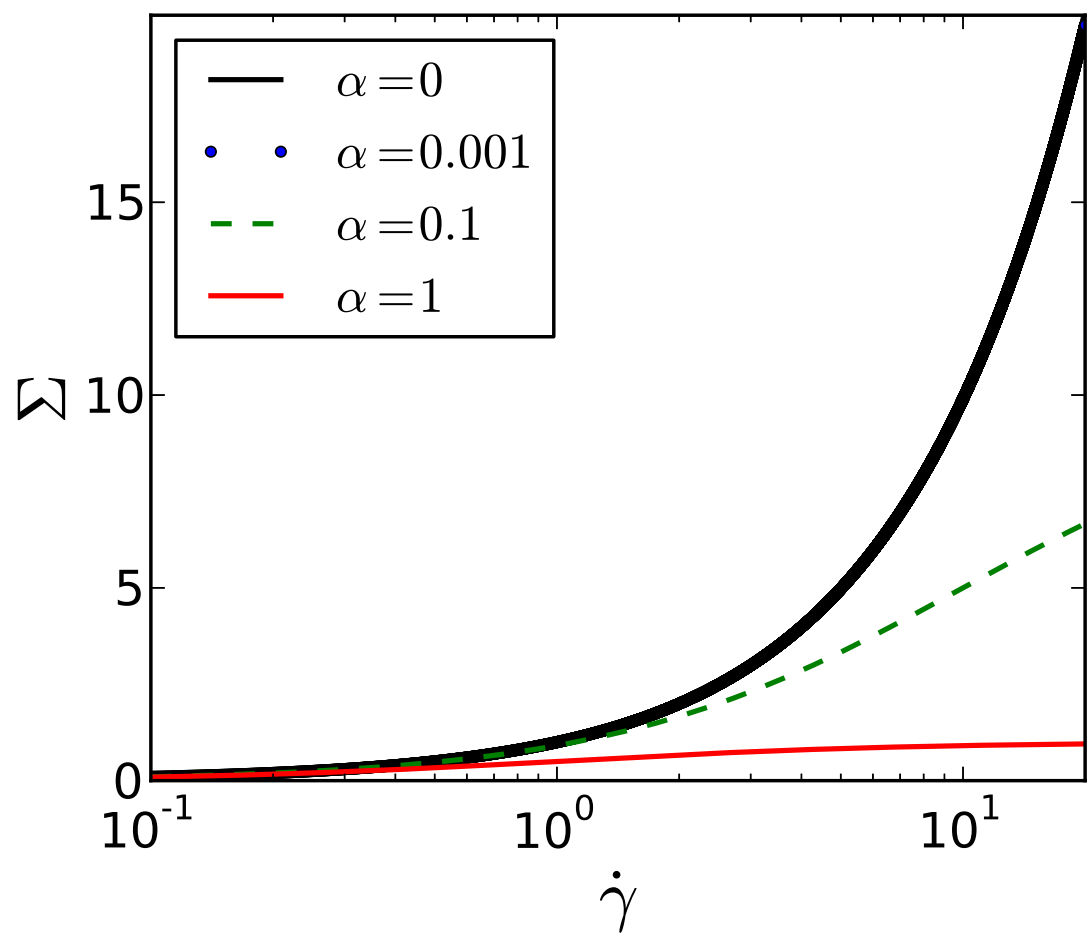

